\title{
Investigation of the role of VHL-HIF signaling in DNA repair and apoptosis in zebrafish
}

\author{
Hyejeong Rosemary Kim¹, Kirankumar Santhakumar ${ }^{2}$, Eleanor Markham ${ }^{1}$, Davide \\ Baldera $^{1}$, David Greenald ${ }^{5}$, Helen E. Bryant ${ }^{3}$, Sherif F. EI-Khamisy ${ }^{4}$ and Fredericus \\ J. van Eeden ${ }^{1}$ \\ ${ }^{1}$ Bateson Centre/BMS, Firth Court, University of Sheffield, Sheffield S10 2TN, UK \\ ${ }^{2}$ Department of Genetic Engineering, SRM Institute of Science and Technology, Kattankulathur 603203, India \\ ${ }^{3}$ Department of Oncology \& Metabolism, The Medical School, Sheffield S10 2RX, UK \\ ${ }^{4}$ Department of Molecular Biology and Biotechnology, Firth Court, University of Sheffield, Sheffield S10 2TN, UK \\ ${ }^{5}$ Centre for Discovery Brain Sciences, University of Edinburgh, Chancellor's Building, Edinburgh EH16 4SB, UK \\ Correspondence to: Fredericus J. van Eeden, email: f.j.vaneeden@sheffield.ac.uk \\ Keywords: Hif; Vhl; DNA repair; apoptosis; chemo/radio-resistance \\ Received: August 14, $2019 \quad$ Accepted: February 17, $2020 \quad$ Published: March 31, 2020
}

Copyright: Kim et al. This is an open-access article distributed under the terms of the Creative Commons Attribution License 3.0 (CC BY 3.0), which permits unrestricted use, distribution, and reproduction in any medium, provided the original author and source are credited.

\section{ABSTRACT}

pVHL is a tumor suppressor. The lack of its function leads to various tumors, among which ccRCC (clear cell renal cell carcinoma) has the most serious outcome due to its resistance to chemotherapies and radiotherapies. Although HIF promotes the progression of CCRCC, the precise mechanism by which the loss of VHL leads to tumor initiation remains unclear. We exploited two zebrafish vhl mutants, vhl and vIl, and Tg (phd3:: EGFP) ${ }^{i 144}$ fish to identify crucial functions of Vhl in tumor initiation. Through the mutant analysis, we found that the role of pVHL in DNA repair is conserved in zebrafish VII. Interestingly, we also discovered that Hif activation strongly suppressed genotoxic stress induced DNA repair defects and apoptosis in vIl and brca 2 mutants and in embryos lacking ATM activity. These results suggest the potential of HIF as a clinical modulator that can protect cells from accumulating DNA damage and apoptosis which can lead to cancers and neurodegenerative disorders.

\section{INTRODUCTION}

In humans, mutations in the pVHL protein, a tumor suppressor protein, predispose patients to Von Hippel Lindau (VHL) disease, a rare form of dominantly inherited cancer syndrome. The patients suffer from recurrent cysts and tumors that can develop into malignant tumors in multiple tissues and organs throughout their lifetime. These include hemangioblastomas of retina and the central nervous system, cysts in pancreas and kidneys, pheochromocytoma and clear cell renal cell carcinoma (ccRCC). Among these, ccRCC is an important cause of death in the VHL patients. It is difficult to be diagnosed at early stages when the tumors are localized and can be treated by surgery, and the advanced forms of tumor are extremely resistant to chemotherapies and radiation therapies. pVHL is also mutated in a majority of cases of the sporadic form of ccRCC, indicating that VHL is a major tumor driver in ccRCC $[1,2]$.
$\mathrm{pVHL}$ is best known as a negative regulator of Hypoxia Inducible Factor (HIF) transcription factors that play crucial roles for the adaptation of cells to the hypoxic environment. HIF transcription factors consist of two subunits, oxygen sensitive $\alpha$ and constitutively present $\beta$. In a normal oxygen tension environment, HIF $\alpha$ is hydroxylated by oxygen sensing enzymes, Prolyl Hydroxylases (PHDs) that use oxygen as substrates. Once it is hydroxylated, HIF $\alpha$ is degraded by E3 ubiquitin ligase complex where pVHL is a substrate recognition subunit. However, when cells are exposed to hypoxia, the inactivation of PHD enzymes lead to HIF $\alpha$ stabilization, its translocation into the nuclei and the formation of functional heterodimers with HIF $1 \beta$ subunit. HIF heterodimers bind to the HIF responsive element (HRE) in their target genes to activate genes that are involved in the adaptation process in hypoxia such as angiogenesis, erythropoiesis, glucose uptake, glycolysis, cell proliferation and cell survival [3, 4]. 
In the absence of VHL, regardless of the environmental oxygen tension, HIF signaling is constantly activated mimicking hypoxic conditions. Tumors are often hypoxic at their core and the activated HIF is known to be advantageous for the survival of tumor cells and to be associated with poor prognosis in various cancers including ccRCC [5]. Understanding the molecular pathways of ccRCC led to the development of a few target therapies. Therapies that target angiogenesis have been a logical approach to treat ccRCC, but only have temporary effect that are accompanied by significant side effects. Alternative approaches may be developed through deeper understanding of the role of VHL.

There are a multitude of HIF independent functions of $\mathrm{pVHL}$ that could be potentially targeted for ccRCC treatment, although these are not as intensively investigated as HIF regulation. These include microtubule stabilization and maintenance of the primary cilium, regulation of the extracellular matrix formation, and control of cell senescence and apoptosis, transcriptional regulation [6] and DNA repair [7, 8]. The mutations in pVHL that cause pheochromocytoma retain the ability to regulate HIF degradation, indicating that HIF independent roles of pVHL are important for tumorigenesis [9]. Also, activating mutations in either HIF $1 \alpha$ or HIF $2 \alpha$ that are associated with ccRCC development, have not been identified in ccRCC patients [10]. In contrast to earlier studies with ccRCC cell lines and tumor xenograft studies which suggested the role of HIF $1 \alpha$ as a tumorsuppressor, more recent mouse transgenic model with constitutive activation of HIF $1 \alpha$ demonstrated the role of HIF $1 \alpha$ in the early ccRCC lesion formation, such as renal cyst and clear cell morphology. Nevertheless, the transgenic animal did not reproduce ccRCC faithfully with invasion and metastasis [11-13] indicating that HIF independent roles of pVHL could also be important for the ccRCC development $[14,15]$.

Besides accumulated HIF, genomic instability is one of characteristics of ccRCC. Evidence suggests that the accumulation of DNA lesions in our body is associated with cancer development and aging [16]. Indeed, inherited mutations in the genes that play important roles in the DNA repair pathways predispose patients to cancer development or premature aging [17]. For example, mutations in one allele of the $B R C A 2$ gene that promotes double strand break repair (DSB) by homologous recombination (HR), increase susceptibility to breast and ovarian cancer, and mutations in ATM that is crucial for DNA repair and cell cycle control upon DNA damage, cause Ataxia -Telangiectasia (A-T) syndrome that is characterized by the very high risk of malignancy, radiosensitivity and progressive ataxia. Heterozygous individuals have an increased risk of cancer $[18,19]$. In line with this, Metcalf et al. demonstrated that $\mathrm{pVHL}$ is required for DSB repair in ccRCC cell lines, implicating the role of pVHL in DNA damage repair as a cause of the genomic instability in ccRCC [7]. In this study, pVHL was hypothesized to work together with ATM upon DNA damage to trigger downstream signaling for the recruitment of DNA repair proteins at the site of DNA damage.

Similarly, Scanlon et al. demonstrated that there was an accumulation of DNA damage in $V H L$ defective ccRCC cell lines compared to $V H L$ complemented cells [8]. There was also downregulation of genes that regulate DSB repair and mismatch repair (MMR) in the ccRCC cells, that may explain the increase in the DNA damage seen. The authors suggest that the VHL deficient cells activate processes that are similar to those in the cells exposed to hypoxia. It was speculated that the downregulation of DNA repair genes in ccRCC cell lines is due to the activation of HIF2 rather than HIF1, since ccRCC cells expressing only HIF2 exhibit the same gene expression profile as that of the cells expressing both HIF transcription factors, i. e. downregulated DNA repair genes. This study also demonstrated the increased sensitivity of ccRCC cells to PARP inhibitor likely because of the DSBR defect in the ccRCC cells.

Therefore, it is clear that the role of VHL in the DNA repair is associated with ccRCC development. However, there are fundamental discrepancies in the above two studies. Although both studies were performed using ccRCC cell lines, Metcalfe et al. reported that the role of VHL in DSBR is HIF independent, whereas Scanlon et al. suggested that the defects in the DNA repair in the $v h l$ mutant cells are similar to those in the cells exposed to hypoxia and they are likely to involve HIF2 transcription factor. This is the limitation of the studies using isolated cells: the cells accumulate mutations in the adaptation process and become different from the tumours they are originated from, although cell lines have certainly been extremely valuable in identifying cancer drugs (e. g., PARP inhibitors) [20]. In addition, the drug responses in the cell lines often cannot be recapitulated in human clinical trials [21, 22]. Therefore, to consolidate the findings in the cell lines we need a whole organism model in which cells maintain their contextual environment and do not undergo the adaptation process in the laboratory environment. Besides, already transformed cancer cell lines are difficult to use for studying the cancer initiation.

More importantly, ccRCC is notorious for its resistance to chemotherapeutic reagents; therefore, it can be speculated that if there is a defect in the DNA repair in the ccRCC as the above two papers suggest, the tumor cells will be extremely sensitive to, rather than resistant to, the DNA damaging reagents further emphasizing the limits of cell lines for studies. We hypothesize that even if the DNA repair function of VHL is important for the initiation of ccRCC development, there must be mechanisms that compromise the effect of the loss of its function and provide resistance to therapies as ccRCC progresses. Indeed, the activation of HIF $1 \alpha$ is known to be associated 
with the resistance of various tumors to chemo and radio therapies by mechanisms that are extremely diverse and complex, e.g. [23]. Similarly, HIF $1 \alpha$ was shown to provide the radioresistance in hypoxic mice mesenchymal stromal cells by upregulating DNA repair proteins [24]. pVHL is also known to regulate p53 that is another crucial transcription factor in the adaptation of cells in response to genotoxic stress and its malfunction provides various tumours with resistance to chemo and radio therapies [25]. Therefore, using zebrafish as a whole organismal model, we aim to understand the role of HIF dependent and independent role of VHL in DNA repair and apoptosis and the role of VHL/HIF in the p53 regulation in response to genotoxic stress.

Zebrafish provides an excellent high throughput vertebrate model system. Nearly $70 \%$ of human genes have orthologous genes in zebrafish and when only disease related genes are considered, around $82 \%$ of genes are associated with at least one zebrafish orthologue [26]. Zebrafish also provides advantages over higher vertebrate models such as fecundity, in vitro fertilisation and easy genetic manipulation. Due to a genome duplication event, there are two zebrafish VHL orthologues, vhl and vll (vhl like). Previously we reported the role of $v h l$ in the HIF regulation and the null zebrafish $v h l$ mutant mimics Chuvash polycythemia in human [27-29]. In this report, we generated a mutant for the $v h l$ paralogous gene, $v l l$, by zinc finger endonuclease and investigated the function of $v h l$ and $v l l$ in the DNA repair. We took advantage of $\operatorname{Tg}(p h d 3: \because E G F P)^{i 144}$ reporter line which expresses a high level of EGFP in the absence of functional $v h l$ but a minimal level of EGFP in the presence of one wild type allele of $v h l$ [30]. We used $v h l+/-; \operatorname{Tg}(p h d 3:: E G F P)^{i 144}$ fish as a unique tool to study in vivo genomic instability using the $v h l$ gene as a "sentinel", since cells express a high level of EGFP when the remaining wild type $v h l$ is lost.

Interestingly the function of human VHL in HIF regulation and DNA repair seems to be partially segregated into zebrafish Vhl and V1l respectively, Hif regulation in Vhl and DNA repair in Vll. We found that the role of Vll in the DNA repair is Hif independent. Surprisingly however, we identified a role of Hif in the promotion of DNA repair and protection of embryos from apoptosis when embryos were exposed to genotoxic stress. Upregulated Hif suppresses not only the DNA repair defects in the $v l l$ mutants but also the defects in the brca2 mutants and the embryos in which ATM function is inhibited. We hope our results will provide a better understanding of ccRCC development and open up great possibilities for the HIF activators to be exploited for the treatment of disorders associated with DNA repair defects. We think our vhl; $v l l$ double mutants can also provide an especially useful system for drug discovery to identify drugs that could overcome the resistance of tumour cells to chemo- and radio therapies.

\section{RESULTS}

\section{Genetic knock out of $v h l$ paralogue $v l l$, by target specific endonuclease}

There are two zebrafish $v h l$ orthologues, $v h l$ and $v l l$ ( $v h l$ like). The function of zebrafish $v h l$ has been well characterized and documented [27, 28]. The function of its paralogue $v l l$, however, has not been reported yet. Therefore, to fully appreciate the function of Vhl in zebrafish, we created a genetic knock-out of $v l l$ by a zinc finger nuclease. The mutant allele lacks 23 nucleotides around transcription starting site including ATG and it is expected to be functionally null (Figure 1A). Sequence analysis revealed that zebrafish Vhl shares $70 \%$ of similarity with human VHL whereas zebrafish Vll shares $52 \%$ of similarity (Figure 1B). As we previously reported, $v h l$ mutant embryos can survive up to $9 \mathrm{dpf}$, and the mutant embryos show typical hypoxic responses such as increased blood vessel formation, upregulated erythropoiesis and hyperventilation [27]. On the contrary, vll mutant embryos are fertile and fully viable, and show no morphological signs of Hif upregulation (Figure 1C).

When we examined the expression of key Hif target genes by qPCR in the $v l l-/-$ mutant embryos, in spite of the lack of visible phenotypes, there was a modest level of upregulated Hif target genes in the vll-/- mutants. Furthermore, there was a remarkable and more than additive upregulation of HIF target genes in the vhl-/-; $v l l-/-$ double mutants (Table 1). The characteristics of $v h l$ mutants by Hif upregulation, such as the reduced usage of yolk and slowdown in growth, were also more enhanced in $v h l-/-; v l l-/-$ double mutants in comparison to $v h l-/-$ single mutants (Figure 1C). We concluded that, although $v l l$ is not required for Hif regulation in the presence of $v h l$, it plays an important role in Hif regulation in the absence of $v h l$, sharing a functional redundancy with $v h l$.

Previously we found that one of the Hif hydroxylases, phd3, is a direct target of Hif, with HREs identified in its promoter [31, 32], and its expression most dramatically reflects the activity of Hif signaling. Accordingly, we used a BAC engineered phd3 reporter line that expresses EGFP under the phd3 promoter as a tool to measure the level of HIF activation [30]. As expected, the phd3 transgenic vhl homozygous mutant embryos expressed a very high level of EGFP reflecting Hif activation. The expression of EGFP in vll-/-; Tg (phd3::EGFP) $)^{i 144}$ mutant embryos, however, was indistinguishable from that of wild type. This is not in agreement with the modest upregulation of $p h d 3$ expression in $v l l-/-$ embryos in our qPCR data. When we repeated qPCR with rps 29 as a reference gene instead of previous $\beta$-actin, the modest upregulation of $p h d 3$ expression was no longer observed in vll-/-; $T g$ (phd3:: $E G F P)^{i 144}$ mutant embryos (Supplementary Table 1). When we examined the $v h l-/-;$ vll-/-; Tg (phd3:: EGFP) 
${ }^{i 144}$ double mutants, there was an augmented expression of EGFP transgene in comparison to $v h l-/-$ single mutants (Figure 1C and Supplementary Table 1), which confirms the redundant role of $v l l$ and $v h l$ in Hif regulation.

\section{Zebrafish $v l l-/-$ mutant fish are more susceptible to loss of heterozygosity $(\mathrm{LOH})$ in the vhl locus}

Interestingly, in heterozygous $v h l+/-$ mutants, the expression of $\operatorname{Tg}(p h d 3:: E G F P)^{i 144}$ is very low whereas $v h l$ / homozygous mutants express a very high level of EGFP, indicating that one copy of $\mathrm{Vhl}$ is sufficient to suppress the expression of Hif in the $v h l+/-$ heterozygous mutants. While we were examining the $v h l+/-; v l l-/-$ mutant adult fish, we came across very bright EGFP expressing cells in the mutant fish that were not observed in $v h l+/-; v l l+/+$ fish. This meant that there was an increased rate of spontaneous LOH of $v h l$ locus in the $v l l-/-$ mutants in comparison to that in wild type fish (Figure 2A and 2B; quantified in 2C).

We also examined $v l l-/-$ embryos to see whether there is an increase in the spontaneous LOH at the vhl locus. Unlike in adults, however, LOH events in the $v h l$ locus were much rarer in the early embryos. Nevertheless, we found a statistically significant increase in the percentage of $v l l-/-$ embryos that showed at least one LOH event from $7 \%(n=220)$ to $19 \%(n=215)$, when we compared a large number of $v h l+/-; \operatorname{Tg}$ (phd3:: EGFP) ${ }^{i 144}$ and $v h l+/-v l l-/-; \operatorname{Tg}(p h d 3: \because E G F P)^{i 144}$ embryos. The number of LOH cells per positive embryo was also higher in $v l l-/-$ embryos (Figure $2 \mathrm{E}$ and $2 \mathrm{~F}$ ).

From this observation, we realized that $v h l+/$ heterozygous $\operatorname{Tg}$ (phd3::EGFP) ${ }^{i 144}$ transgenic fish could be an excellent tool to study genomic instability. When the cells experience any genotoxic stress which introduces strand breaks or other damage in the DNA of the $v h l$ locus, the cells that repair the damage perfectly, e. g. by HR, will remain EGFP negative. On the other hand, if, for instance, a DNA break is repaired by mechanisms that introduce errors or if there are defects in the DNA repair pathways, the cells will lose the remaining wild type allele of $v h l$ and will express a very high level of EGFP (Figure 2D). Therefore, we reasoned that $v l l$ might be required for maintaining genomic stability and the role of human VHL in DNA repair might be conserved in zebrafish $v l l$.

\section{Zebrafish vll-/- mutant embryos increase LOH at vhl locus in response to ionizing irradiation}

Using $\operatorname{Tg}(p h d 3:: E G F P)^{i 144}$ as a tool to study the genomic instability, we treated $v l l-/-$ mutant embryos with a high level of X-ray to introduce DSBs. We hypothesized that if $v l l$, like the human $V H L$ gene, is required for the DNA DSB repair by HR upon DNA damage the repair of irradiation induced DNA damage in the $v h l$ locus in $v l l-/-$ mutants would not be as efficient as in wild type embryos, and there will be an increase in the number of EGFP positive $v h l-/-$ cells in the $v l l-/-$ mutant. We irradiated vhl+/-; vll-/- ; Tg (phd3:: EGFP) ${ }^{i 144}$ transgenic embryos with X-rays at $1 \mathrm{dpf}$ and examined the $\mathrm{LOH}$ at $v h l$ locus at $5 \mathrm{dpf}$. Its $\mathrm{LOH}$ rate was compared with that of irradiated $v h l+/-; v l l+/+$ embryos. We found that the $\mathrm{LOH}$ rate in the $v h l$ locus in the $v l l-/-$ mutant embryos was much higher than that in $v l l+/+$ wild type embryos indicating the role of $v l l$ in accurate DNA repair (Figure 3A).

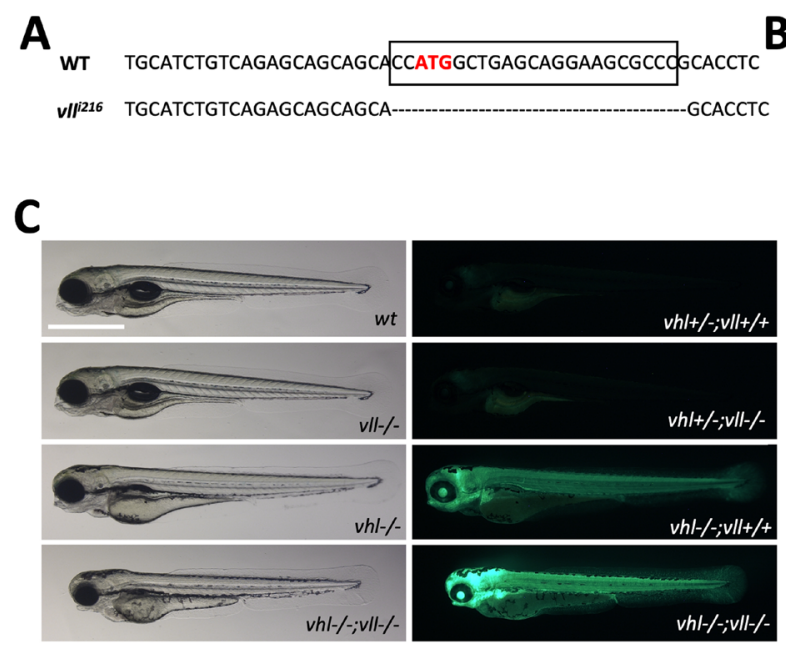

B $\begin{aligned} & \text { hvhl } \\ & \text { mvhl } \\ & \text { zvhl } \\ & \text { zvll }\end{aligned}$
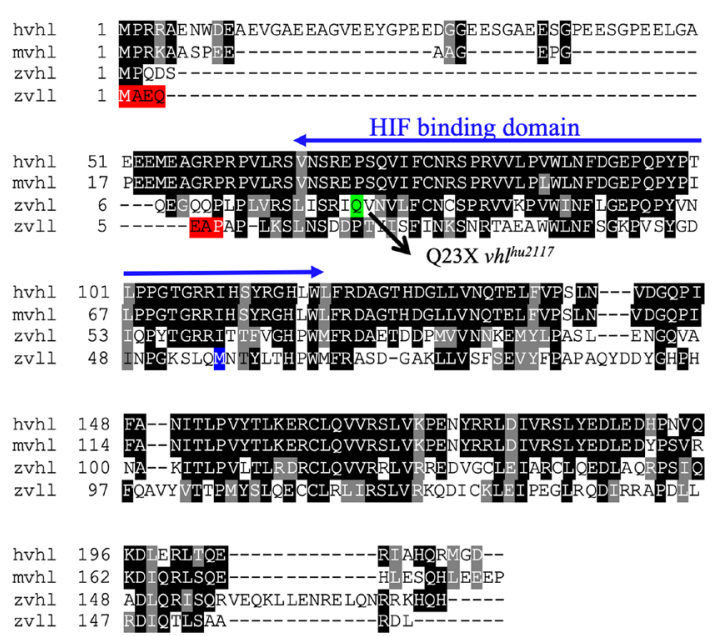

Figure 1: A $v \boldsymbol{l l}$ mutant allele, $\boldsymbol{v} \boldsymbol{l l}^{216}$, is generated by a zinc finger nuclease. (A) The mutant allele harbors 23 nucleotide deletion around translational starting site. (B) Zebrafish Vll shares 52\% homology with human VHL. The seven amino acids that are lost in $v l l^{216}$ are highlighted in red. Even if the next available ATG (highlighted in blue) were to be used as an alternative translational start site, it would lead to a protein that lacks most of the predicted Hif binding domain. (C) Since $p h d 3$ (egln3) expression most dramatically reflects the increased Hif expression, we used $\operatorname{Tg}(p h d 3:: E G F P)^{i l 44}$ transgenic line as a Hif signaling readout. Zebrafish $v l l-/-$ embryos do not show any morphological phenotype and the $\operatorname{Tg}$ (phd $3:$ EGFP) ${ }^{i l 44}$ expression in the mutants was identical to that in the wild type embryos. Reflecting the role of Vll in Hif regulation in the absence of Vhl, there was an enhanced GFP expression in the vhl-/-; vll-/- double mutant embryos in comparison to that in $v h l-/-$ embryos. Scale bar: $1 \mathrm{~mm}$. 
Table 1: The qPCR results reveal the modest role of Vll and the synergistic effect of the loss of Vhl and Vll in the Hif regulation

\begin{tabular}{lcccc}
\hline Gene Name & Gene Symbol & $\boldsymbol{v l l}$ vs $\boldsymbol{w t}$ & $\boldsymbol{v h l}$ vs $\boldsymbol{w t}$ & vhl; $\boldsymbol{v l l}$ vs $\boldsymbol{w t}$ \\
\hline egl-9 family hypoxia-inducible factor 3 & egln3 & 3.51 & 30.20 & 281.99 \\
vascular endothelial growth factor Aa & vegfaa & 4.11 & 4.89 & 26.98 \\
aminolevulinate, delta-, synthase 2 & alas2 & 1.82 & 11.60 & 15.41 \\
MAP kinase interacting serine/threonine kinase $2 \boldsymbol{b}$ & mknk2b & 3.53 & 6.56 & 14.24 \\
GATA binding protein1a & gatala & 1.99 & 2.79 & 3.63 \\
\hline
\end{tabular}

We performed qPCR to examine the expression level of a few Hif target genes. There was a modest upregulation of Hif target genes in $v l l$ mutants indicating the minor role of $v l l$ in Hif regulation in the presence of vhl. However, a striking upregulation of Hif target genes was observed in $v h l-/-; v l l-/-$ mutants suggesting the redundant role of $v h l$ and $v l l$ in the Hif regulation. The values are fold changes, and they are means of three technical and two biological replicates.

Next, we used the CRISPR/Cas9 system that specifically targets the $v h l$ locus, to introduce a targeted DNA DSB in $v h l$. Using EGFP expression in the $T g$ (phd3:: EGFP) ${ }^{i l 44}$ as a readout for $\mathrm{LOH}$, we injected a single gRNA against $v h l$ into 1-cell stage $v h l+/-$; $v l l-/-$ embryos and examined the $\mathrm{LOH}$ at $5 \mathrm{dpf}$ with the equally injected $T g$ (phd $3:: E G F P)^{i l 44}$; vhl+/-; vll+/+ embryos as their control. Similar to the results from X-ray treatment, there was an increased number of EGFP positive cells in the $v l l-/-$ mutants in comparison to $v l l+/+$ wild type embryos, indicating that accurate DNA repair is defective in the $v l l$ mutant (Figure 3B).

Loss of heterozygosity can be due to various mechanisms either local mutations or more significant changes like loss of an entire chromosomal region. We investigated efficiency of mutagenesis with CRISPR/Cas9 system that targets not only vhl but also another gene, Androgen Receptor $(A R)$. We injected guide RNAs targeting $A R / v h l$ into $v l l-l-$ mutants and wild type embryos to quantify mutagenesis rates in the vll- -- mutants in comparison to wild type embryos. After the injection, genomic DNAs were extracted and PCR was performed to amplify the sequences around the target sites. We carried out deep sequencing of the PCR products from injected samples. This revealed that there was no significant increase in the mutation frequencies, or in the size of indels in the $v l l-/-$ mutants in both $A R$ and vhl targeting CRISPR injected embryos (Supplementary Figure 1). This suggests that increased LOH in the vll-/embryos might be due the loss of larger regions of the chromosome or complex rearrangements of chromosomes that cannot be detected by PCR methods [33].

To exclude the possibility that $v l l$ protects embryos from the DNA damage induction rather than it being involved in DNA repair, we examined the expression of $\gamma \mathrm{H} 2 \mathrm{AX}$ which is one of the earliest responses to DNA damage after the irradiation. We irradiated $v h l+/-$ and $v h l+/-; v l l-/-$ embryos at $1 \mathrm{dpf}$ and fixed them immediately after the X-ray treatment. The fixed embryos were examined for their $\gamma \mathrm{H} 2 \mathrm{AX}$ foci formation. This revealed that embryos of all genotypes showed equally distributed formation of $\gamma \mathrm{H} 2 \mathrm{AX}$ foci after the X-ray treatment suggesting that DNA damage was equally introduced in all genotypes (Supplementary Figure 2).

\section{$v h l-/-$; vll-/- double mutants are protected from $\mathrm{X}$-ray induced apoptosis}

We wondered whether $v h l$ and $v l l$ have functional redundancy in their DNA damage repair role, and wanted to investigate the possibility that $v h l$ might play a major role in the DNA repair, in the same way as in their Hif regulation. Unfortunately, however, we cannot use our $\operatorname{Tg}(p h d 3:: E G F P)^{i l 44}$ line as a tool to study the role of $v h l$ in genomic instability because all the cells in the $v h l$ mutants already lack both alleles of wild type $v h l$ and express a very high level of EGFP. Therefore, we decided to examine the sensitivity of $v h l-/-$; vll-/- double mutants to genotoxic stress compared with that of $v l l-/-$ mutant. We reasoned that if $v h l$ and $v l l$ are both required for the DNA repair, the double mutant would be extremely sensitive to the genotoxic stress.

We pair mated $v h l+/-;$ vll-l-; $T g$ (phd $3::$ EGFP) ${ }^{i 144}$ fish to expose $v h l-/-$; $v l l-/-$ double mutants to X-ray treatment. When the collected embryos were irradiated at $1 \mathrm{dpf}$ and examined at $5 \mathrm{dpf}$, we found that around a quarter of embryos were extremely well protected from the X-ray induced cell death. We separated these protected embryos from the rest and examined them under the fluorescent microscope. Surprisingly, it turned out that all the protected embryos were $v h l-/-; v l l-/-$ double mutants with a very high level of EGFP expression (Figure 4A-4D). This was in strong contrast to our speculation that double mutant would be extremely sensitive to the genotoxic stress. TUNEL staining confirmed a much reduced number of apoptotic cells in the double mutants in comparison to their siblings (Figure 4E-4G).

\section{Hif activation protects embryos from genotoxic stress induced apoptosis}

We then wanted to identify what makes $v h l-/-; v l l-$ / - double mutants resistant to the X-ray treatment. The 
most obvious effect of the double mutation is upregulation of Hif signaling. Therefore, we sought to upregulate Hif signaling independently of Vhl and Vll to test whether Hif upregulation could protect embryos from cell death induced by genotoxic stress. We employed a cancer therapeutic reagent Camptothecin (CPT), which induces DSBs in replicating cells [34-36]. Embryos from $v h l+/-$; vll-/- pair mating were treated with $20 \mathrm{nM} \mathrm{CPT}$ at $32 \mathrm{hpf}$ overnight. The CPT treated embryos were examined for their sensitivity to CPT at 5dpf. All the embryos treated
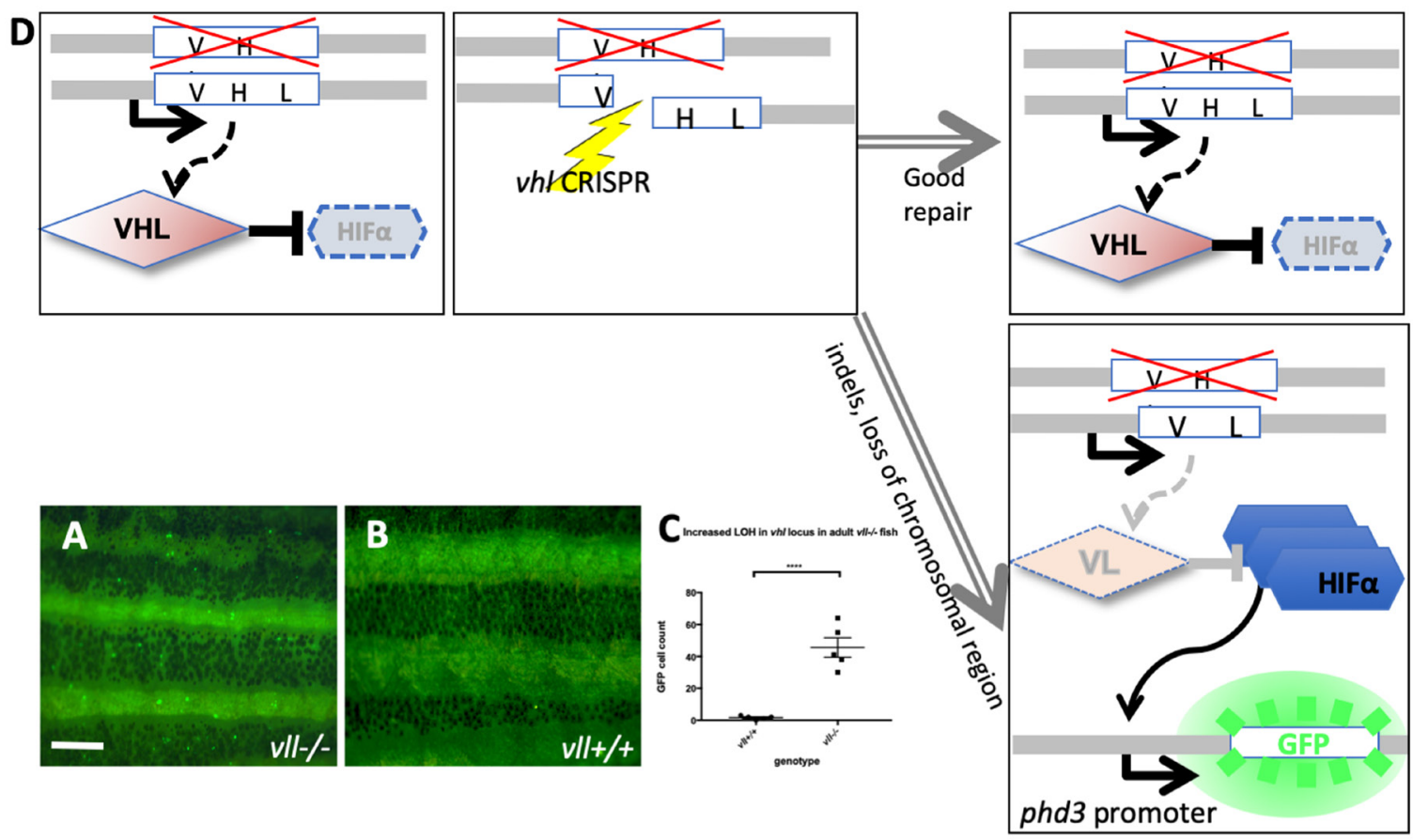

$\mathbf{E}$

\section{Compare to wild-type, a greater faction of vll-/- embryos show spontaneous LOH events}

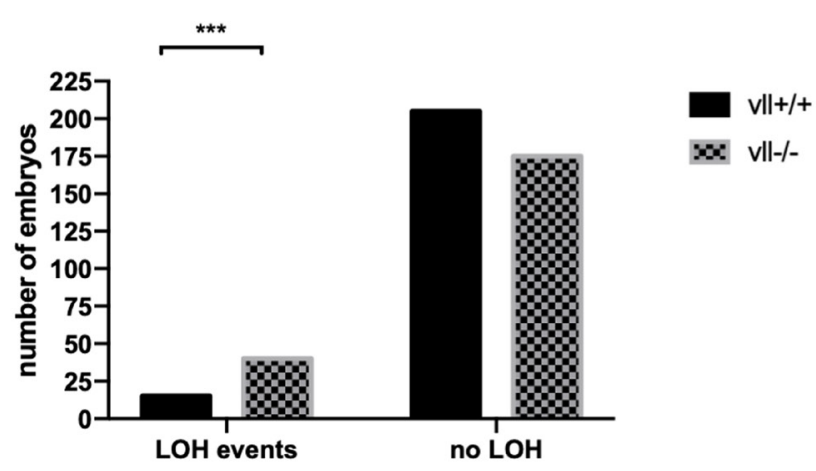

$\mathbf{F}$

\section{Compared to wild-type, vll--- show a higher number of spontaneous LOH cells per embryos}

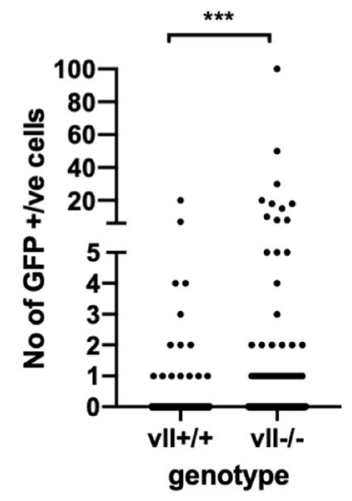

Figure 2: The adult $\boldsymbol{v l l}-/-$ mutant fish are more susceptible to LOH in the $\boldsymbol{v h l}$ locus. (A and B) When we examined 6 months old $v l l-/-$ mutant fish, there was an increased number of spontaneous LOH in the $v h l$ locus in $v l l-/-$ mutant fish in comparison to $v l l+/+$ wild type fish. The increased LOH in $v l l-/-$ mutant fish was quantified in $(\mathbf{C}){ }^{* * * *} p<0.0001$, unpaired $t$-test. Scale bar: $1 \mathrm{~mm}(\mathbf{D})$ Schematic diagram illustrating the principle of our reporter system that if DNA damage is introduced into the remaining wildtype $v h l$ allele, for instance by a CRISPR, in the $v h l+/-: T g(p h d 3:: E G F P)^{i l 44}$ fish in response to the genotoxic stress and the damage is well repaired and no frameshift is introduced, the cells will remain EGFP negative. However, if the DNA damage introduced into the wild type allele is not repaired properly, the cells will lose heterozygosity and express very bright EGFP expression. (E) we also examined the spontaneous $\mathrm{LOH}$ at the $v h l$ locus in $v l l-/-$ mutant embryos. Although the number of LOH events was much lower than that in adults and a majority of embryos did not have any $\mathrm{LOH}$ events, we found a statistically significant increase in the number of embryos that experienced a LOH event in $v l l-/$ - embryos in comparison to wild type embryos. ${ }^{* * * *} p<0.0001$, Chi-square (and Fisher's exact) test. (F) There was an increase in the number of EGFP positive cells in $v l l-/-$ embryos in comparison to wild type embryos. ${ }^{* * *} p<0.001$, unpaired $t$-test. 
with CPT, except $v h l-/-; v l l-/-$ double mutants that express a high level of EGFP, died by $5 \mathrm{dpf}$ with severe cell death (Figure 5A and 5B) confirming our previous observation. Further, when a parallel batch of embryos were treated with Hif activator (JNJ-42041935, 100 $\mathrm{uM}$ ) for 8 hours prior to CPT treatment, all the embryos, regardless of their $v h l$ genotype, were protected from CPT treatment induced death, suggesting that the protection of $v h l-/$-; vll-/- double mutants from CPT treatment was indeed due to elevated HIF activation (Figure 5C and 5D). To confirm these data further, we treated wild type embryos with CPT at $20 \mathrm{nM}$, with or without pretreatment with Hif activator. This revealed that all the wild type embryos treated with CPT alone died by $5 \mathrm{dpf}$ (Figure 5E), whereas the majority of embryos survived when they were treated with HIF activator prior to CPT treatment (Figure 5F and Supplementary Figure 3). Hif activator proved to be very effective at a wide range of concentrations: the protective effect has been seen at the concentration as low as $25 \mathrm{uM}$ and as high as $200 \mathrm{uM}$ without any sign of toxicity. Chemical Hif activators function by inhibiting cellular hydroxylases and could therefore have additional effects. To further show that the protection of the embryos is due to upregulated Hif, we injected constitutively active forms of Hifl $\alpha$ and Hif $2 \alpha$ to induce a high level of Hif activation, instead of chemically activating Hif. This also showed that all the Hif injected embryos were protected from CPT induced apoptosis, confirming the specificity of chemical Hif activator (Figure 5G, compare to $5 \mathrm{E}$ ). When we examined apoptosis in the Hif activator treated embryos by TUNEL staining after CPT treatment at $10 \mathrm{nM}$ concentration, there was a significant reduction in the TUNEL positive cells in the Hif activator treated embryos in comparison to the untreated wild type embryos (Supplementary Figure 3).

\section{p53 expression is decreased in the vhl-/-; $v l l-/$ double mutants and in $v h l-/-$ mutants in comparison to their siblings}

Previously, a direct role of VHL in p53 stabilisation and transactivation has been reported, with diminished p53 protein levels and activity in the absence of $v h l$ [37]. Since we observed reduced apoptosis in the $v h l-/-$; vll-/- mutants and Hif activated embryos in response to genotoxic stress, we questioned whether the reduction in apoptosis in these embryos is due to the downregulation in p53 expression. According to the previous studies
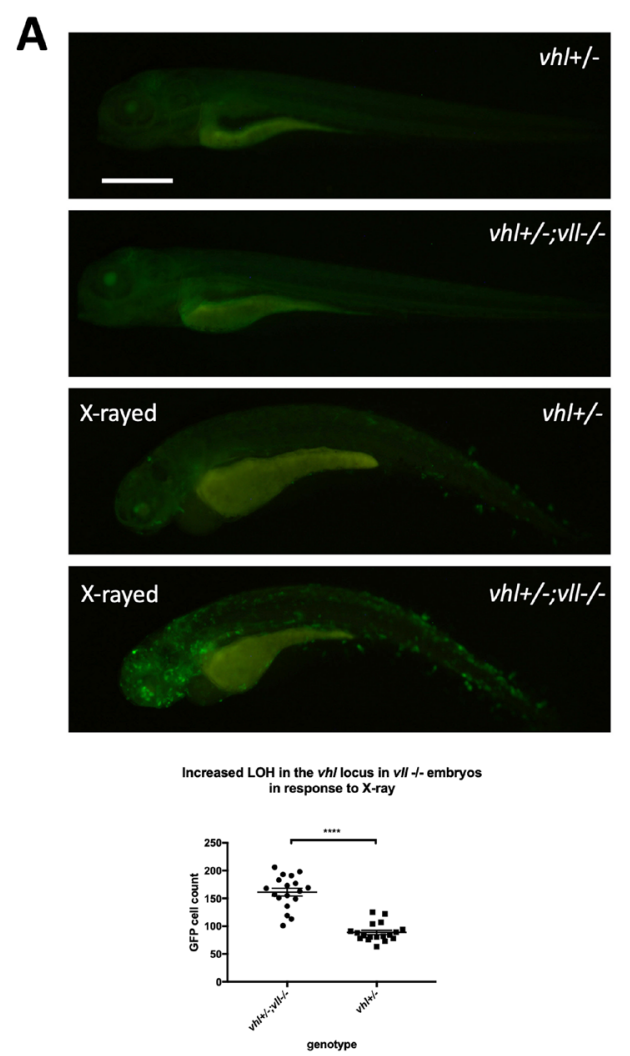

\section{B}

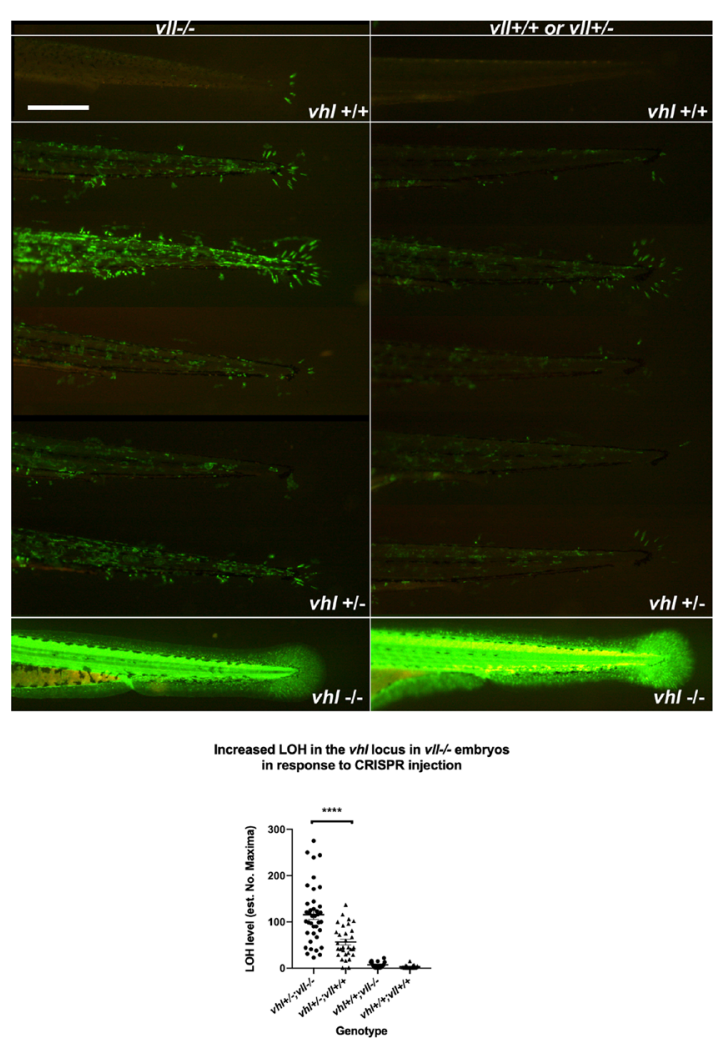

Figure 3: There is an increase in $\mathrm{LOH}$ in $v l l-/-$ embryos in response to $\mathrm{X}$-ray treatments and CRISPR injection. (A) There was an increased LOH in the $v h l$ locus in $v l l-/-$ embryos in response to X-ray treatments. ${ }^{* * * *} p<0.0001$, unpaired $t$-test. (B) Injection of gRNA/Cas9 against $v h l$ locus induced increased LOH at the $v h l$ locus in $v l l-/-$ embryos in comparison to $v l l+/+$ wild type embryos. B is a composite image of 14 individual tail images, white lines separate different genotypes. ${ }^{* * *} p<0.001$, one way ANOVA; Holm-Sidak's multiple comparison test. Scale bars: $0.5 \mathrm{~mm}$. 
in zebrafish and cell culture systems, full length p53 is induced immediately in response to X-ray treatment to remove the cells with excessive DNA damage beyond repair and ensure the cells do not progress through the cell cycle and proliferate. A short form $(\Delta 113 p 53)$ that is a direct target of full length p53 and transcribed from the C-terminus of intron 4 of full length p53, is induced much later than the full length p53 to promote DNA DSB repair and to antagonise the pro-apoptotic activity of full length p53 [38].

Considering the suppressed apoptosis phenotype observed, we speculated that full length of p53 would be downregulated in the $v h l-/-; v l l-/-$ double mutants. We $\gamma$-rayed embryos from $v h l+/-; v l l-/-$ pair mating and performed q-PCR against full length p53 and the short form of $\mathrm{p} 53$. There was a dramatic reduction in both isoforms of p53 expression in the vhl-/-; vll-/- mutant embryos in comparison to their siblings (Figure 6A and 6B). p53 suppression in the $v h l-/-$; vll-/- mutants is likely to occur due to the role of Hif in $\mathrm{p} 53$ regulation rather than the direct role of $\mathrm{Vhl}$ in p53 regulation as Roe et al. [37] suggests, since Hif activator treated embryos also have reduced apoptosis.

To further confirm that the protection of embryos from DNA damage induced apoptosis in the Hif activated embryos may be due to downregulation in p53, we treated embryos from $v h l+/-$; vll-/- incross with $10 \mathrm{nM}$ CPT at 32hpf overnight and the induced levels of p53 and $\Delta 113$ p53 protein were examined by Western Blot in $v h l-/-; v l l-/-$ embryos and in their siblings. This revealed
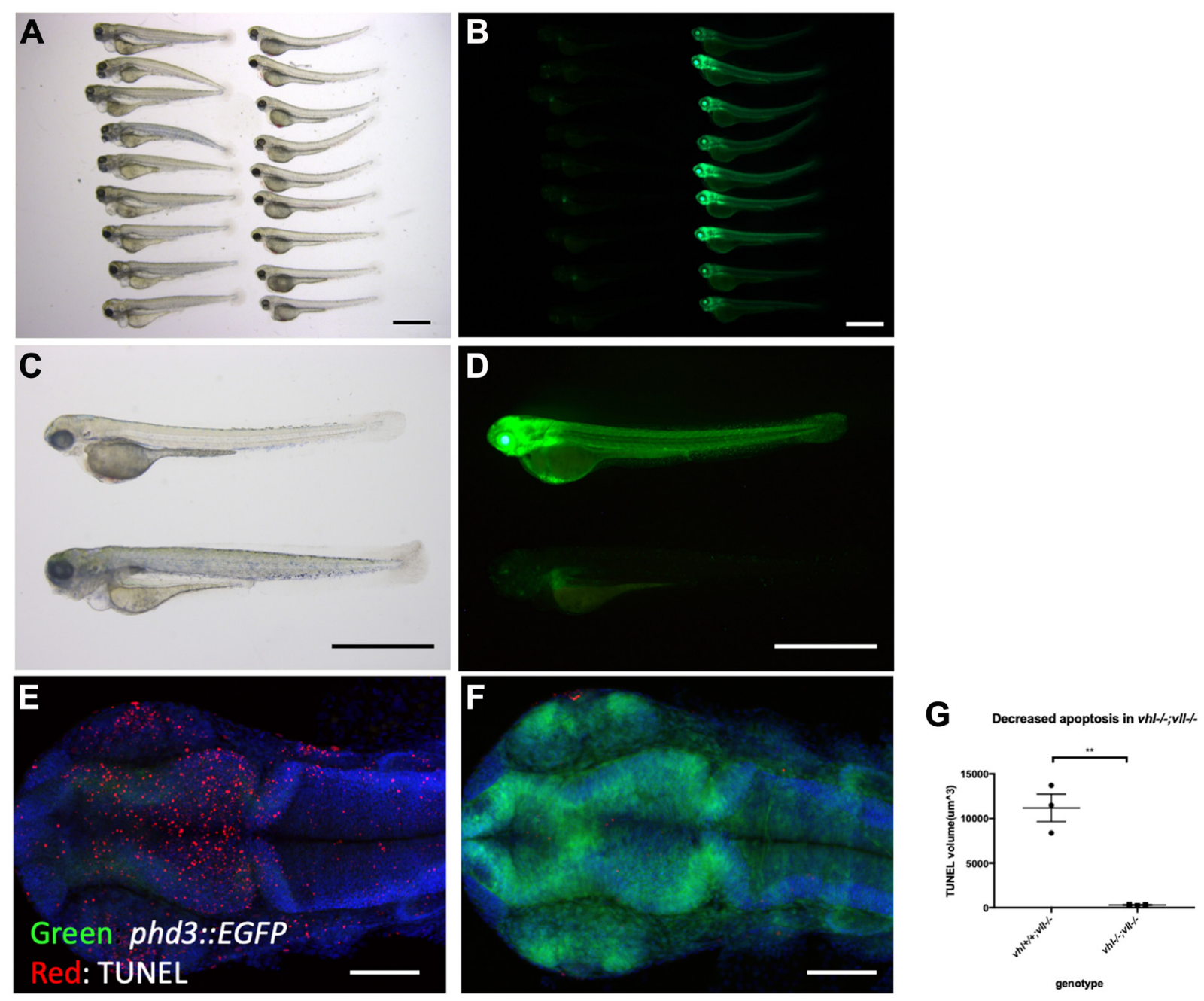

Figure 4: $v \boldsymbol{h l}-/-$; $\boldsymbol{v l l}-/$ - double mutants are protected from X-ray induced apoptosis. (A-D) The embryos from $v h l+/-$; vll-/- pair mating were collected and the embryos were exposed to X-ray treatment at $1 \mathrm{dpf}$. Then the treated embryos were examined at $5 \mathrm{dpf}$. There were clear differences in response to X-ray treatment; majority of embryos were severely affected by X-ray treatment whereas around $25 \%$ of the embryos were well protected without much sign of cell death. We classified these embryos and looked at them under the fluorescent microscope. This revealed that all the protected embryos were EGFP positive $v h l-/-; v l l-/-$ double mutants. (E and F) The embryos were collected from the $v h l+/-$; $v l l-/-$ pair mating, and then exposed to X-ray at $1 \mathrm{dpf}$. The embryos were fixed 3 hours post X-ray and we analysed the cell death in the head region with TUNEL. This showed extremely decreased TUNEL positive cells in the $v h l-/-$; $v l l-/-$ double mutants in comparison to their siblings. (G) Quantification of TUNEL stain. $n=3,{ }^{* *} p<0.01$, unpaired $t$-test. Scale bars: 1 $\mathrm{mm}(\mathrm{A}-\mathrm{D})$ and $0.2 \mathrm{~mm}(\mathrm{E} \& \mathrm{~F})$. 
that the induction of both isoforms of p53 was clearly attenuated in $v h l-/-; v l l-/-$ embryos, which is consistent with our qPCR data (Figure 6C).

To investigate if a reduced p53 level in the vhl-/-; vll-/- double mutants may suffice to suppress apoptosis in the mutants, we investigated the effect of p53 downregulation on the apoptosis, using p53 morpholino injection. When we injected an efficient p53 morpholino (Supplementary Figure 4) into the embryos that were collected from $v h l+/-; v l l-/-$ pair mating and treated them with $10 \mathrm{nM}$ CPT, we observed all the injected embryos were protected from CPT induced apoptosis regardless of their genotype (Figure 7B), to a similar level as in Hif activator treated embryos (Figure 7C), in contrast to uninjected controls (Figure 7A) in which only the $v h l-/$; vll-/- double mutants are protected from CPT treatment; quantified in Figure 7D. These data are consistent with the idea that double mutants can be protected from genotoxic stress due to a low level of p53 expression. We wondered whether Hif activation leads to suppression of apoptosis by additional mechanisms other than downregulating p53. We found that there was a further reduction in the CPT induced apoptosis in p53 morphant when the morphant was pre-treated with Hif activator before the CPT treatment. This suggests Hif plays a protective role not only through the downregulation of p53 but also independent of p53. Similarly, p53 knock down enhanced the Hif activation provided protection in response to CPT treatment, suggesting that Hif suppresses the p53 induction but does not fully eliminate its function (Supplementary Figure 5).

\section{Hif activation protects embryos from genotoxic stress induced DNA damage}

The results clearly demonstrate the role of Hif in the suppression of apoptosis, probably through downregulation of $\mathrm{p} 53$. There are two scenarios that can explain these observations, either lack of p53 activation prevent cells with severe DNA damage from undergoing apoptosis, and consequently these cells with accumulated DNA damage will contribute to the cell population of later embryos, or alternatively HIF might have a truly genoprotective role, improving the resilience of the genome to DNA damage, thereby reducing the p53 activation. To test whether Hif activation plays a role in the genoprotection, we decided to test its effect on the increased DNA damage in the $v l l$ mutants. We treated vll-/- mutants with HIF activator for at least 2 hours prior to X-ray treatment at $24 \mathrm{hpf}$. These embryos were heterozygous for $v h l$ and carried $T g$ (phd3::EGFP) ${ }^{i 144}$ to allow the detection of $\mathrm{LOH}$ levels for $v h l$, as described
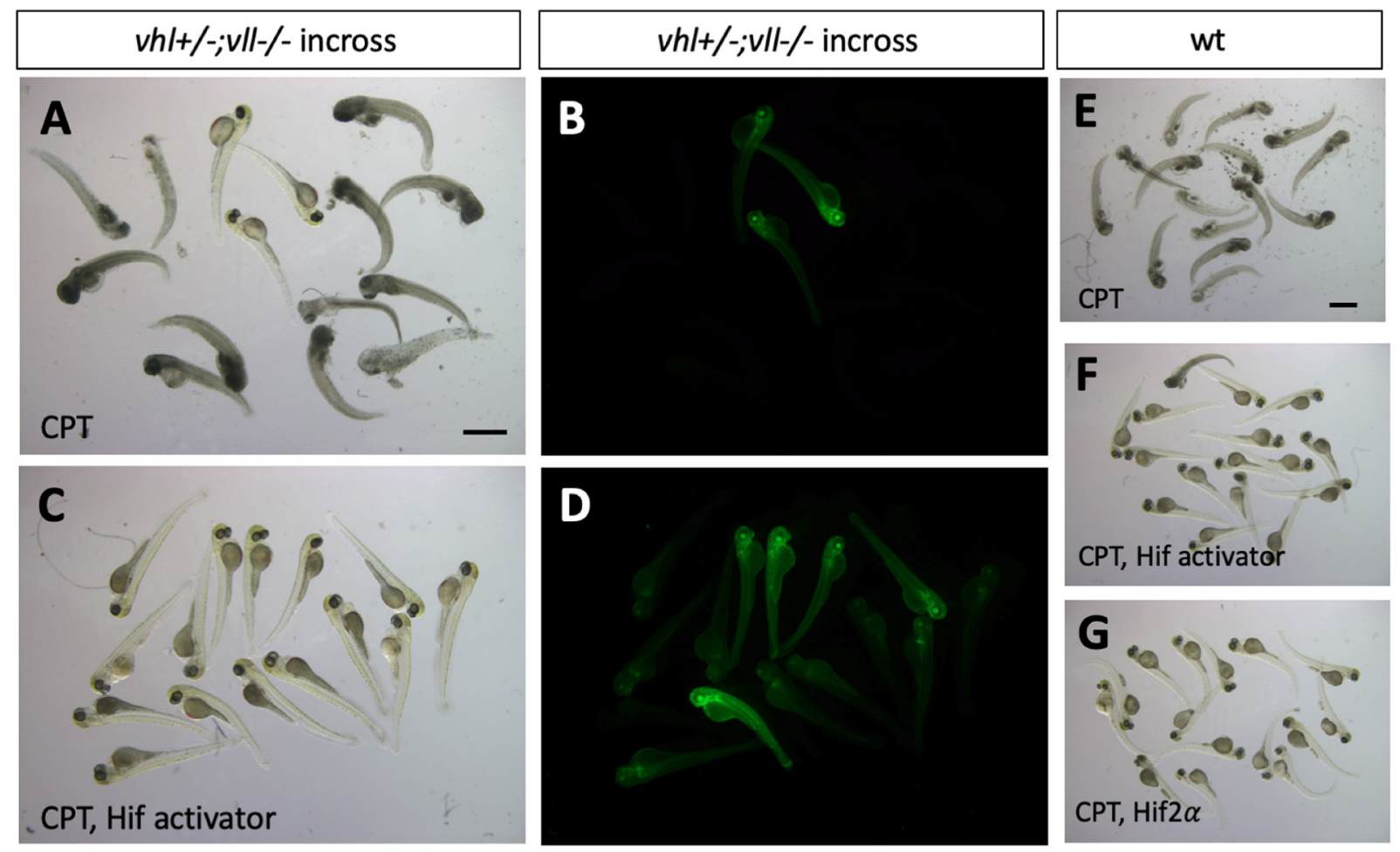

Figure 5: Hif activation protects embryos from CPT induced apoptosis. (A and B) The embryos from $v h l+/-$; $v l l-/-$ pair mating were treated with $20 \mathrm{nM} \mathrm{CPT}$. The majority of embryos did not survive until $5 \mathrm{dpf}$ except a few embryos. All the embryos that survived were EGFP positive $v h l-/-$; $v l l-/-$ double mutants. (C and D) When the embryos were treated with Hif activator for 8 hours prior to the CPT treatment, all the embryos became EGFP positive and survived until $5 \mathrm{dpf}$. (E) When wild type embryos were treated with CPT, the embryos did not survive at $5 \mathrm{dpf}$. (F) However, most of embryos that are treated with Hif activator prior to CPT treatment survived at 5 dpf. (G) The embryos injected constitutively active Hif2 $\alpha$ also survived at $5 \mathrm{dpf}$. Scale bars: $1 \mathrm{~mm}$. 
previously. We would expect that if cells with damaged DNA fail to undergo apoptosis due to lack of p53 function, more cells with LOH might be visible per embryo, but if Hif has a role in protecting cells from DNA damage, the $\mathrm{LOH}$ rate will be reduced. The embryos were examined for their LOH at $5 \mathrm{dpf}$. This revealed that $v l l-/-$ mutant fish treated with Hif activator had significantly decreased LOH (Figure 8B and 8D; quantified in $8 \mathrm{E}$ ), suggesting that Hif itself has a role in the protection of embryos from DNA damage and that the role of Vll itself in DNA repair is likely to be Hif independent. In addition, Hif activator treated embryos looked a lot healthier than untreated embryos, which is consistent with the results from CPT treatment. To quantify the latter we used embryonic eye size as an easily measurable "surrogate" for overall health, and found it to be significantly increased (compare Figure $8 \mathrm{~A}$ and $8 \mathrm{C}$; quantified in $8 \mathrm{~F}$ ).

Then we asked whether downregulation of $\mathrm{p} 53$ has any effect on the DNA repair function of Hif. We injected p53 morpholino into the embryos collected from $v h l+/-$; vll-/-; $\operatorname{Tg}(p h d 3:: E G F P)^{i 144}$ pair mating and treated them with CPT to examine the effect of p53 downregulation on the LOH formation in $v h l$ locus. We counted the number of EGFP positive cells in the $v h l+/-; v l l-/-$ embryos that are induced by CPT treatment and compared with the number of EGFP positive cells in the p53 morpholino injected $v h l+/-; v l l-/-$ embryos. There was no significant difference in the number of EGFP positive cells between p53 morpholino injected and uninjected $v l l-/-$ embryos, indicating the role of Hif in the promoting DNA repair is through p53 independent mechanism (Figure 9A-9C, quantified in 9D). brca2-/- mutants are sensitive to X-ray treatment induced cell death and an elevated Hif level in the vhl-/- mutants alleviates the cell death induced by mutation in brca2

As the precise relationship of Vll to DNA repair is still very poorly understood, we sought to look at the effect of Hif on well-established DNA repair gene mutations. Firstly, we created a brca 2 mutant allele using the CRISPR/Cas9 system. The brca 2 mutant allele for this experiment contained an $83 \mathrm{bp}$ insertion at the amino acid 445 (exon 10) position introducing premature stop. When embryos from brca2+/pair-mating were treated with X-rays, we found that there was increased cell death in homozygous brca2-/- embryos, especially in the central nervous system (CNS) as previously reported [39]. In contrast, the least affected embryos were all either brca $2+/-$ or $b r c a 2+/+$ (Figure 10A). We confirmed the increased apoptosis in the brca2-/- embryos in comparison to their siblings by Acridine Orange staining (Figure 10B).

We then proceeded to check whether elevated Hif activation seen in the vhl-/-; vll-/- mutants can also suppress the increased sensitivity of brca2-/- embryos to genotoxic stress. We generated brca2+/-; vhl+/-; vll-/mutants. The embryos from brca2+/-; vhl+/-; vll-/- in cross were treated with $\mathrm{X}$-rays at $1 \mathrm{dpf}$ and examined for the cell death in the CNS 2 days post X-ray. This revealed that the increased apoptosis in the brca2-/- mutant was suppressed by the presence of $v h l-/-$; vll-/- and that in this background, brca2-/- embryos were indistinguishable from their siblings, suggesting that the elevated Hif can strongly suppress the sensitivity of brca2-/- embryos to $\mathrm{X}$-ray induced apoptosis (Figure 10D).

\section{The Hif activation in the vhl-/-; vIl-/- embryos suppresses the induction of p53 and $\Delta 113 p 53$ in response to DNA damage}
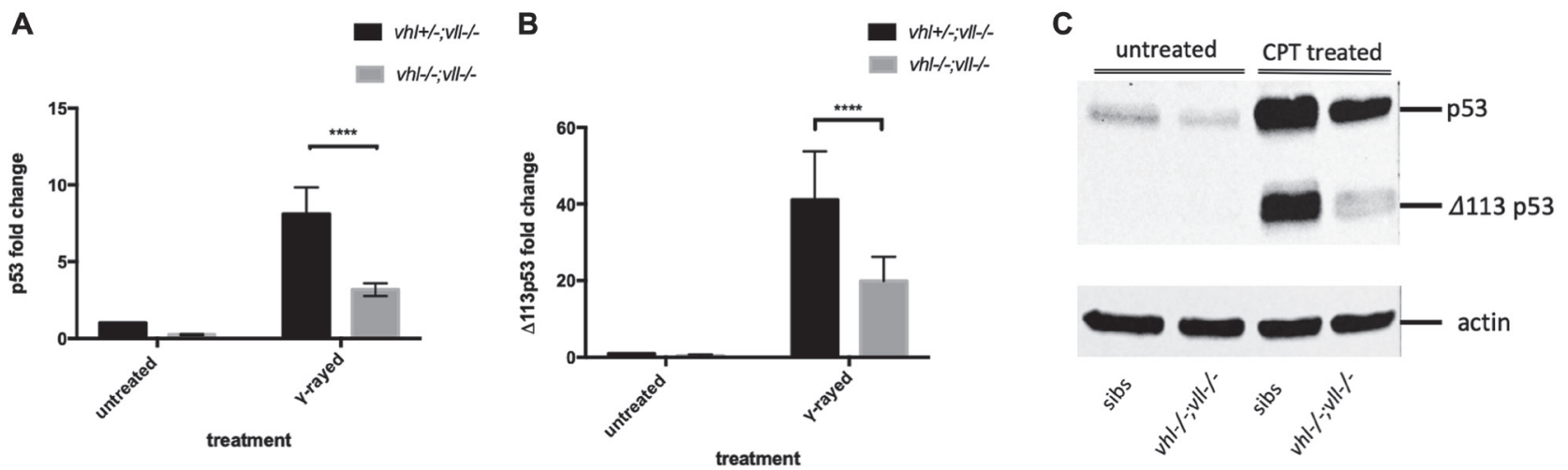

Figure 6: p53 expression in the vhl-/-; vll-/- double mutants is downregulated in comparison to that in their siblings. (A and B) Embryos from $v h l+/-$; vll-/- pair mating were collected and irradiated at $2 \mathrm{dpf}$ with $\gamma$-ray at a dose of 20 gray. q-PCR was performed 24 hours post $\gamma$-ray to quantify the full length of p53 and short form of $\Delta 113 \mathrm{p} 53$. This revealed that there was a significant decrease in both forms of $\mathrm{p} 53$ expression in the $v h l-/-$; $v l l-/-$ embryos in comparison to their siblings. ${ }^{* * * *} p<0.0001$, two way ANOVA test. The statistical analysis was performed with ddCt values. (C) We also treated embryos from $v h l+/-$; $v l l-/-$ incross with $10 \mathrm{nM} \mathrm{CPT}$ and both isoforms of 553 induction was examined in the $v h l-/-;$ vll-/- embryos and in their siblings by Western blot. After treatment, there was a clear reduction in both isoforms of p53 expression in the $v h l-/-$; vll-/- embryos in comparison to their siblings. 
We also treated brca2-/- embryos and their siblings with CPT with or without Hif activator pre-treatment. There was a significant suppression in the CPT induced apoptosis in the brca2-/- embryos when they were pretreated with Hif activator, although not to the same level as that in their siblings. We speculate that the elevation of Hif level by chemical Hif activator treatment is not as powerful as that induced by the loss of Vhl and Vll (Supplementary Figure 6).

In addition, we examined the effect of the loss of brca 2 in the rate of loss of $v h l$ alleles in the brca2-/-; Tg

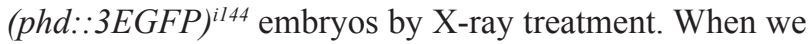
exposed the embryos from brca2+/-; $T g$ (phd::3EGFP) ${ }^{i 144}$ pair mating to a high dose of X-ray, there were a few cells expressing a high level of EGFP in brca2-/- embryos, indicating that these cells had lost both $v h l$ wild type alleles, in contrast to their siblings in which there were hardly any EGFP positive cells. When these embryos were pre-incubated with Hif activator before the X-ray treatment, the number of EGFP positive cells in the brca2/ embryos was profoundly reduced, proving the role of Hif in the protection of brca2-/- embryos from the X-ray induced DNA damage (Figure 10C).

\section{Hif activation abrogates the sensitivity to CPT induced cell death in ATM inhibitor treated embryos}

Another fundamental regulator of the DNA damage response is ATM (ATM serine/threonine kinase), and ATM
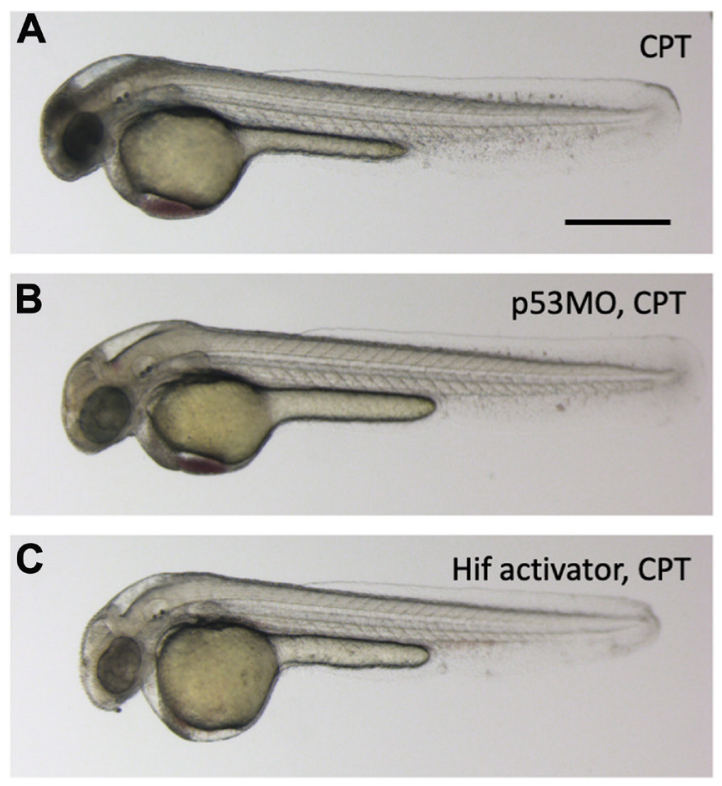

deficient cells are highly sensitive to genotoxic stresses [40]. Patients with mutations in ATM develop A-T syndrome that are characterised by progressive neurodegeneration, increased risk of cancer development, radiosensitivity and immune system impairment. We wondered whether Hif activator can mitigate the effect of the loss of ATM through its role in the anti-apoptosis and DNA repair. We treated wild type embryos with $10 \mathrm{nM}$ CPT with or without pre-incubation with $10 \mu \mathrm{M}$ ATM inhibitor (ATMi). As expected, ATMi treated embryos were highly sensitised to CPT treatment with increased apoptosis in the brain in comparison to ATMi untreated embryos. However, when the embryos were treated with Hif activator in combination with ATMi before CPT treatment, Hif activator treated embryos were well protected from CPT induced cell death (Figure 11A-11C; quantified in 11G). We also observed the protection of ATMi treated embryos from X-ray induced LOH by Hif activator treatment. ATMi treated embryos were highly sensitised to X-ray treatment with increased $\mathrm{LOH}$ at the $v h l$ locus in comparison to ATMi untreated embryos. On the contrary, when the embryos were treated with both Hif activator and ATMi, the increased LOH at the $v h l$ locus was dramatically reduced (Figure 11D-11F; quantified in $11 \mathrm{H})$.

\section{HIF activator treatment does not protect human MRC5 cells from X-ray treatment}

We were very surprised by the strength of the protective effect that Hif conferred in zebrafish embryos

D

\section{p53 knock down efficiently suppresses the CPT induced apoptosis}

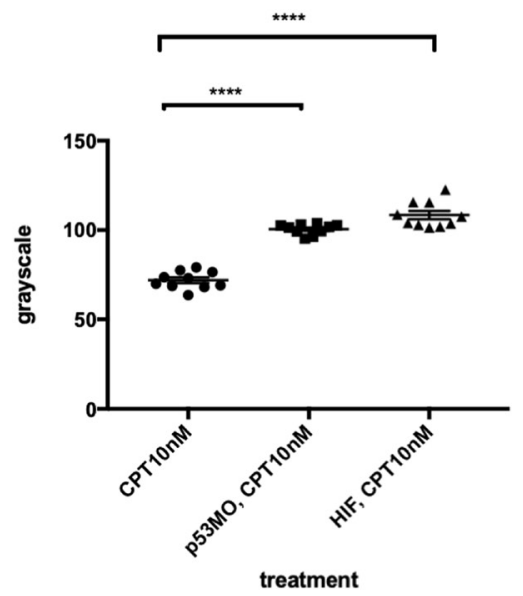

Figure 7: p53 knock down by p53 morpholino injection protects embryos from CPT induced apoptosis. (A) Embryos collected from $v h l+/-$; $v l l-/-$ pair mating were treated with $10 \mathrm{nM} \mathrm{CPT}$. This resulted in severe cell death in the CPT treated embryos with the exception of GFP positive $v h l-/-$; vll-/- double mutants. (B) On the contrary, when p53 morpholino was injected into the embryos prior to $10 \mathrm{nM} \mathrm{CPT} \mathrm{treatment,} \mathrm{all} \mathrm{the} \mathrm{embryos} \mathrm{were} \mathrm{protected} \mathrm{from} \mathrm{CPT} \mathrm{induced} \mathrm{apoptosis.} \mathrm{(C)} \mathrm{The} \mathrm{similar} \mathrm{protection} \mathrm{was} \mathrm{also} \mathrm{observed} \mathrm{in} \mathrm{the}$ embryos treated with Hif activator prior to CPT treatment. Only GFP negative embryos were imaged and quantified for this experiment. (D) The image quantification of levels of apoptosis using gray scale in ImageJ, low gray scale values denote high levels of apoptosis. ${ }^{* * * *} p$ $<0.0001$, one-way ANOVA. Scale bar: $0.5 \mathrm{~mm}$. 
and we were eager to see if it is conserved in cultured human cells. As cancer cells may have defects in p53 or other relevant pathways, we initially chose MRC5 that is the most common human diploid fibroblasts cell line derived from normal lung tissue of a 14 weeks old male fetus. We took MRC5 cells with or without HIF activator (FG4592) at $15 \mathrm{uM}$ concentration and the cells were incubated for 3 hours at $37^{\circ} \mathrm{C}$ followed by X-ray treatment at 5 gray. The cells were fixed 4 hours and 24 hours post irradiation (hpi) and stained with $\gamma \mathrm{H} 2 \mathrm{AX}$ to examine the DNA damage foci. Surprisingly, we observed that there was no statistical difference in $\gamma \mathrm{H} 2 \mathrm{AX}$ formation between the HIF activator treated group and non-treated group at both time points (Figure 12A). In our second experiment, we decided to lower the $\mathrm{X}$-ray dose to 2 gray since we could not observe the resolution of $\gamma \mathrm{H} 2 \mathrm{AX}$ at $24 \mathrm{hpi}$ from the first experiment and tested an alternative cell line, human osteosarcoma U2OS. U2OS cells were treated with HIF activator (JNJ-42041935) at $100 \mathrm{uM}$ concentration and incubated cells for 3 hour before the X-ray treatment at 2 gray. The cells were fixed 30 minutes post irradiation (mpi) and 24 hpi to examine $\gamma \mathrm{H} 2 \mathrm{AX}$ formation. When
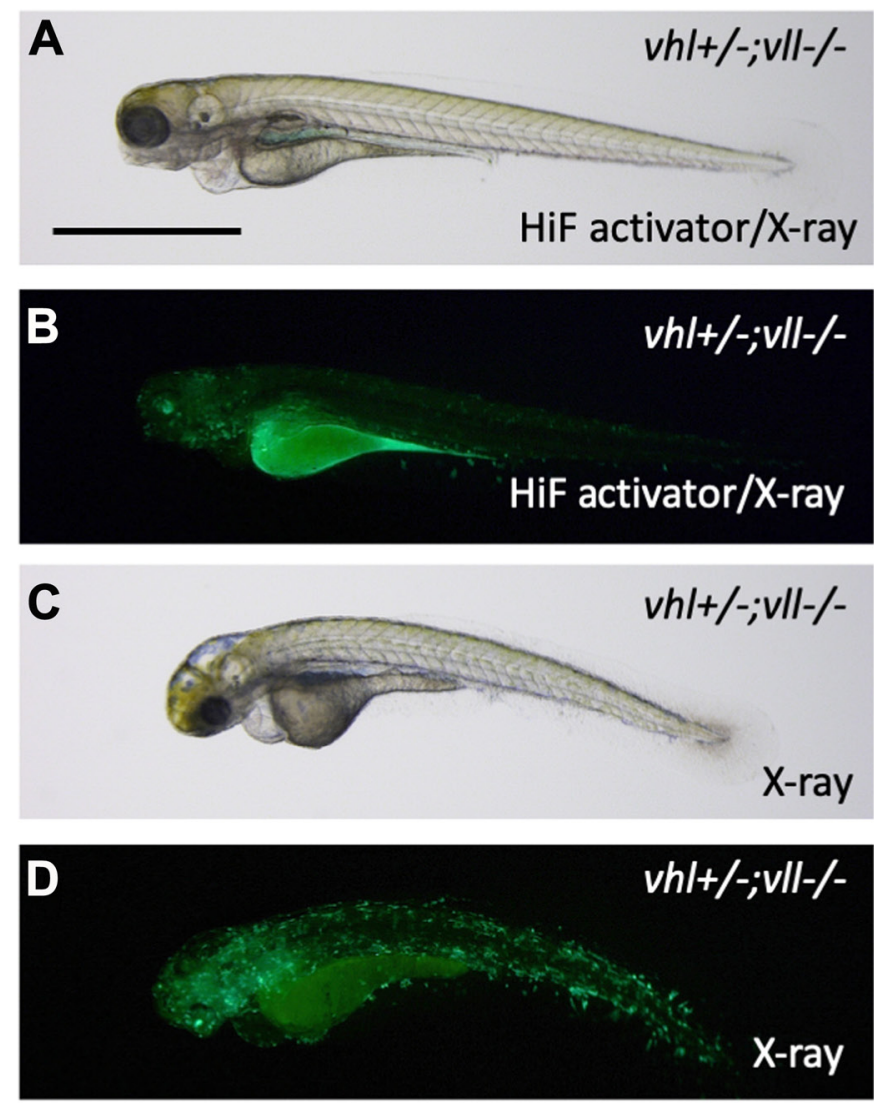

we counted the number of $\gamma \mathrm{H} 2 \mathrm{AX}$ foci, we still could not observe the genoprotective effect of HIF activator treatment at both time points (Figure 12B).

\section{Hif activator treatment does not promote colony formation in the MRC5 cells}

As an alternative readout for Hif mediated protection to DNA damaging treatment in cell culture, we performed a clonogenic survival assay. The MRC5 cells (4000 cells) were seeded a day before the drug treatment. The cells were then treated with HIF activators with or without ATM inhibitor. 3 hours later, CPT was added either at $10 \mathrm{nM}$ or $25 \mathrm{nM}$ concentration. The drugs were washed off after 1 hour incubation. The cells were then left to form colonies in the $37^{\circ} \mathrm{C} \mathrm{CO}_{2}$ incubator. Seven days later, the colony formation was examined. There was decreased number of colonies in the CPT/ATM inhibitor treated cells in comparison to untreated control cells. Unlike in zebrafish, however, in the cell culture studies, we could not find any increase in the survival rates in the HIF activator treated cells in comparison to the non-treated cells (Figure 13).

\section{E Hif activator suppresses LOH in vhl locus in the absence of vll- -}

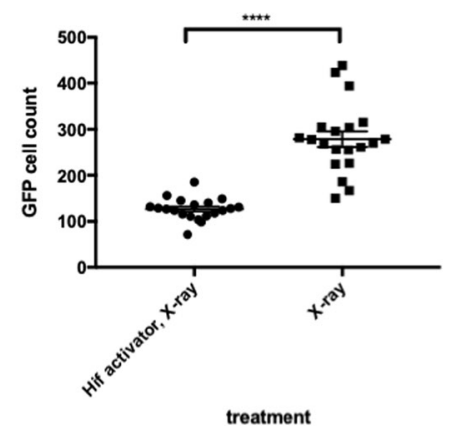

$\mathbf{F}$ Embryos treated with Hif activator are healthier than untreated embryos

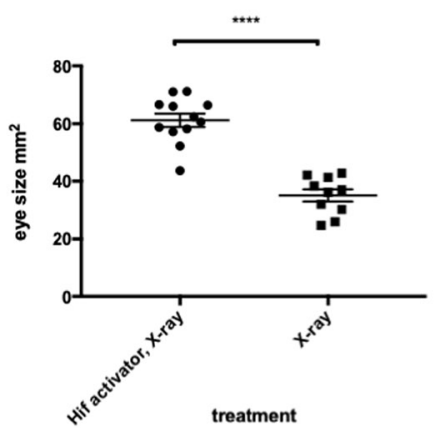

Figure 8: Hif activator treatment protects embryos from X-ray induced DNA damage. (A-D) We investigated the effect of Hif activation on the increased LOH in $v h l$ locus in $v l l-/-$ mutant embryos. When embryos were treated with Hif activator prior to X-ray treatment, the increased LOH in the $v h l$ locus in $v l l-/-$ embryos was significantly reduced (B) in comparison to $v l l-/-$ embryos that were exposed to X-ray without Hif activator treatment (D). (E) The effect of Hif activator on the LOH suppression in the vhl locus was quantified. ${ }^{* * * *} p<0.0001$, unpaired $t$-test. Hif activator treated $v l l-/-$ embryos also looked more healthier (A) than untreated embryos $(\mathrm{C})$. (F) The size of eyes was measured as a surrogate for general health, and quantified in the Hif activator treated and untreated embryos. ${ }^{* * * *} p$ $<0.0001$, unpaired $t$-test. Scale bar: $1 \mathrm{~mm}$. 


\section{DISCUSSION}

ccRCC is one of the most difficult cancers to treat because of its resistance to chemotherapies and radiotherapies. Although a few targeted therapies have improved the survival rates of ccRCC patients to a certain extent, advanced ccRCC still remains incurable. In addition, unlike some other tumor suppressor proteins, pVHL is a highly multifunctional protein that makes it difficult to design target therapies against. Evidently upregulated HIF in ccRCC provides survival advantages to cancer cells. The activation of HIF, however, although necessary for the ccRCC progression, is not sufficient to initiate tumor development [41-43].

According to the mutator phenotype theory in cancer, the rate of random mutations cannot explain the frequency of mutations in cancer, and the mutations in the genes that are essential for the maintenance of genomic stability, including genes that are involved in DNA repair, have been speculated to increase the mutation rates and drive cancer development [44, 45]. In line with this theory, Metcalfe et al. demonstrated that human pVHL is required for the DSBR in the ccRCC cell lines, leading us to speculate that the loss of DNA repair function of VHL might account for the initiation of ccRCC in the VHL patients [7]. We set out to corroborate these data using a whole organismal vertebrate model, zebrafish, to overcome the limitation
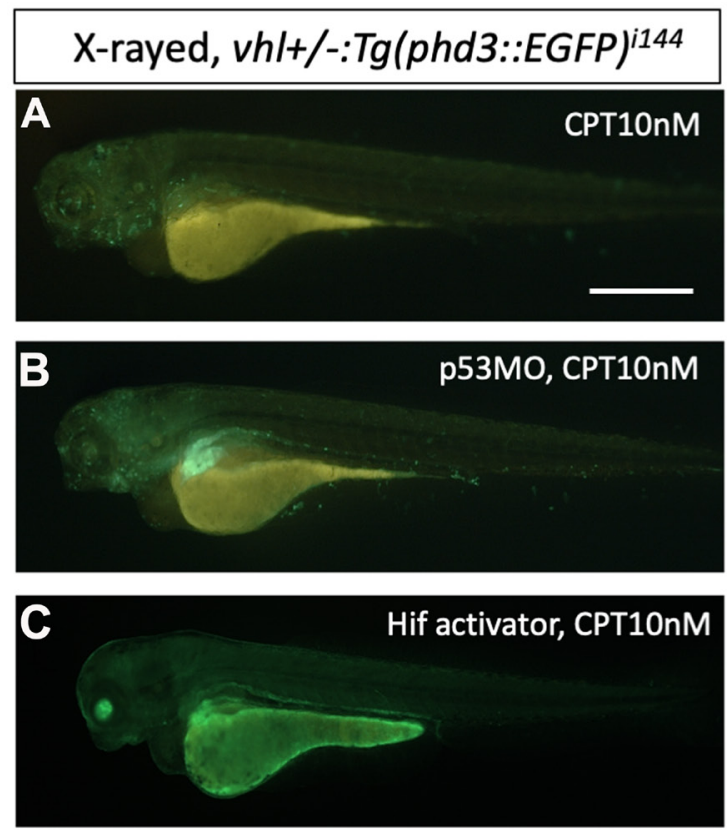

of the already transformed cancer cell lines for the functional studies for VHL.

We found that in zebrafish, among two VHL orthologous genes, the role of VHL in HIF regulation is mainly performed by Vhl, whereas the role of Vll in Hif regulation is very modest. We found that, consistent with the studies in the ccRCC cell lines, vll mutant embryos were more susceptible to genotoxic stress and induced increased LOH in our assay with $T g$ (phd3:: EGFP) $)^{i 144}$ reporter line, indicating the role of VHL in DNA repair is conserved in Vll in zebrafish. However, when we performed deep sequencing around the target sites of CRISPRs against AR and Vhl, the rate of mutation frequencies generated in $v l l$ mutants was not significantly different from $v l l$ wild type embryos. The precise reasons for this remain unclear, perhaps the defects that are observable by the LOH assay may consist of more significant changes to the $v h l$ containing chromosome, e. g. the loss of a chromosome arm, as is thought to occur in VHL patients [33]. As our next gene sequencing assay is based on PCR amplification, such deletion events go undetected. In order to get a better understanding of the genoprotective effect of HIF, it would be highly interesting to look at such chromosomal events, however this would require genome analysis in individual embryonic cells. Such single-cell ploidy analysis is now becoming feasible [46].

We hypothesized that Vhl might have a more dominant role in the DNA repair than its paralogous gene Vll, considering its higher homology with human VHL.

\section{D}

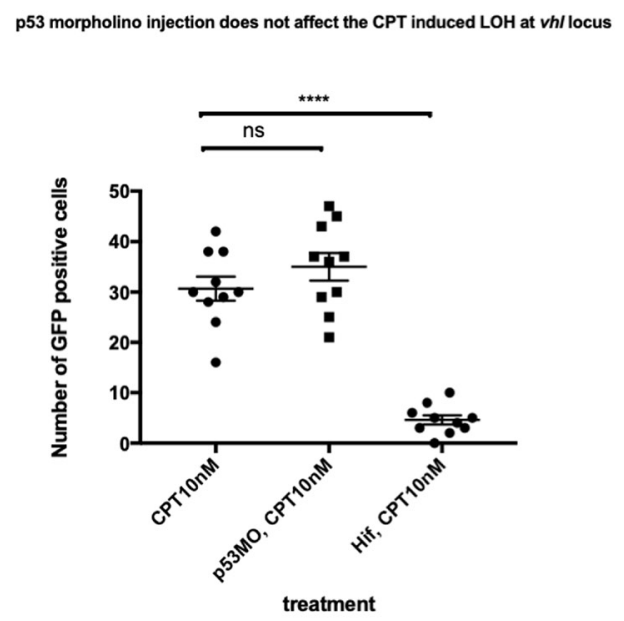

Figure 9: p53 knock down by p53 morpholino does not affect the number of LOH at the $v h l$ locus induced by CPT. (A) The embryos collected from $v h l+/-$; $v l l-/$ pair mating were treated with $10 \mathrm{nM} \mathrm{CPT}$. There was GFP positive cells throughout the CPT treated embryos indicating the LOH in these cells. (B) A similar number of GFP positive cells were observed in the p53 morpholino injected embryos suggesting that the CPT induced LOH formation in these embryos is independent of p53 level. (C) On the contrary, the treatment of Hif activator prior to CPT treatment abolished the LOH formation in the embryos, signifying the genoprotective role of Hif against CPT treatment. (D) The number of EGFP positive cells were quantified using ImageJ ( $n=10$ per treatment). ${ }^{\mathrm{n} s} p>0.05,{ }^{* * * *} p<0.0001$, one-way ANOVA. Scale bar: $0.5 \mathrm{~mm}$. 
Unfortunately, we could not use $\operatorname{Tg}(p h d 3: \because E G F P)^{i 144}$ $\mathrm{LOH}$ reporter assay to study the $\mathrm{LOH}$ in the $v h l$ mutants, since all the cells in the $v h l$ mutants already express a high level of EGFP and there is no wild type $v h l$ allele remaining that can be used as "reporter". We reasoned, however, that $v h l-/-; v l l-/-$ double mutants will have an increased sensitivity to genotoxic stress, if not only $v l l$ but also $v h l$ is involved in the DNA repair pathway. But in marked contrast to our expectation, we found that $v h l-/$; vll-/- double mutant embryos were highly protected from the genotoxic stress with much reduced apoptosis, when the embryos were treated with X-ray and CPT.

Considering the prominent upregulation of Hif in the $v h l-/-; v l l-/-$ double mutants, we then tested whether the elevated Hif in double mutants is responsible for the protection of embryos from the genotoxic stress induced cell death. When we treated embryos with pharmacological
Hif activator prior to X-ray and CPT treatment, Hif activation resulted in the protection of embryos from apoptosis induced by such genotoxic stressors, even in the presence of intact $v h l$ and $v l l$. Injection of dominantly active form of Hif into the newly fertilized embryos also resulted in the protection of embryos from the CPT treatment in the same way as pharmacological activation of Hif results, confirming the role of Hif in the providing embryos with protection against genotoxic stress.

In addition, when pharmacologically activated, Hif suppressed the increased LOH in the $v l l$ mutants. We speculate that this may happen by activating as yet unidentified DNA repair pathways. As a result, in the $v h l-/-; v l l-/-$ double mutants in which there is a maximal level of increased Hif signaling, the DNA repair defect due to the lack of $v l l$ was suppressed by the elevated Hif activation and the embryos were well protected from
A

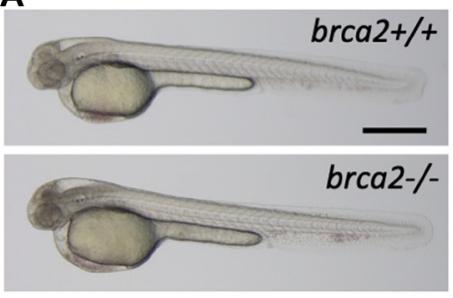

B

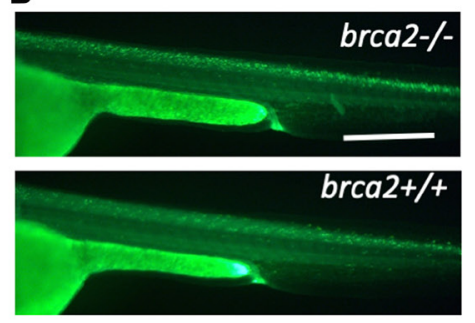

Green: Acridine Orange

C

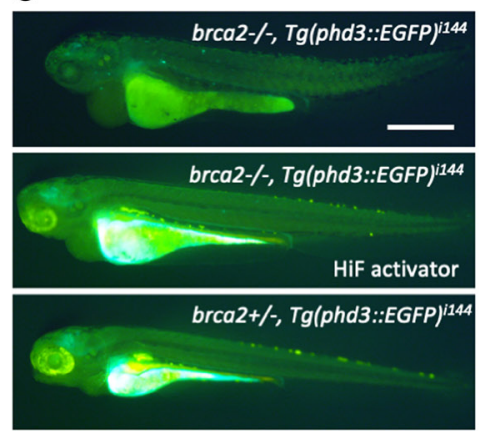

brca2-- embryos are more sensitive to $X$-ray treatment

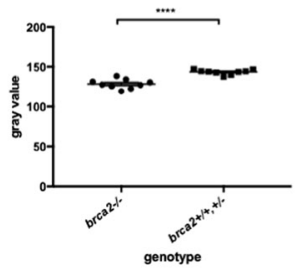

There is an increased apoptosis in brca2-- embryos

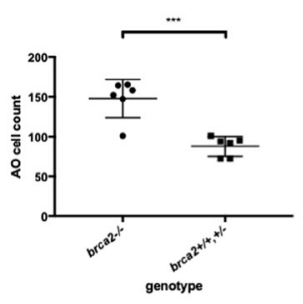

Hif activator significantly reduces $X$-ray induced DNA damage in brca2-- embryos

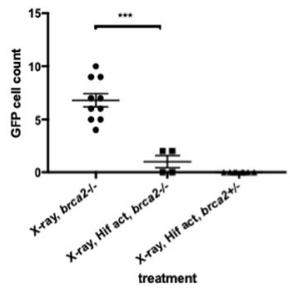

D

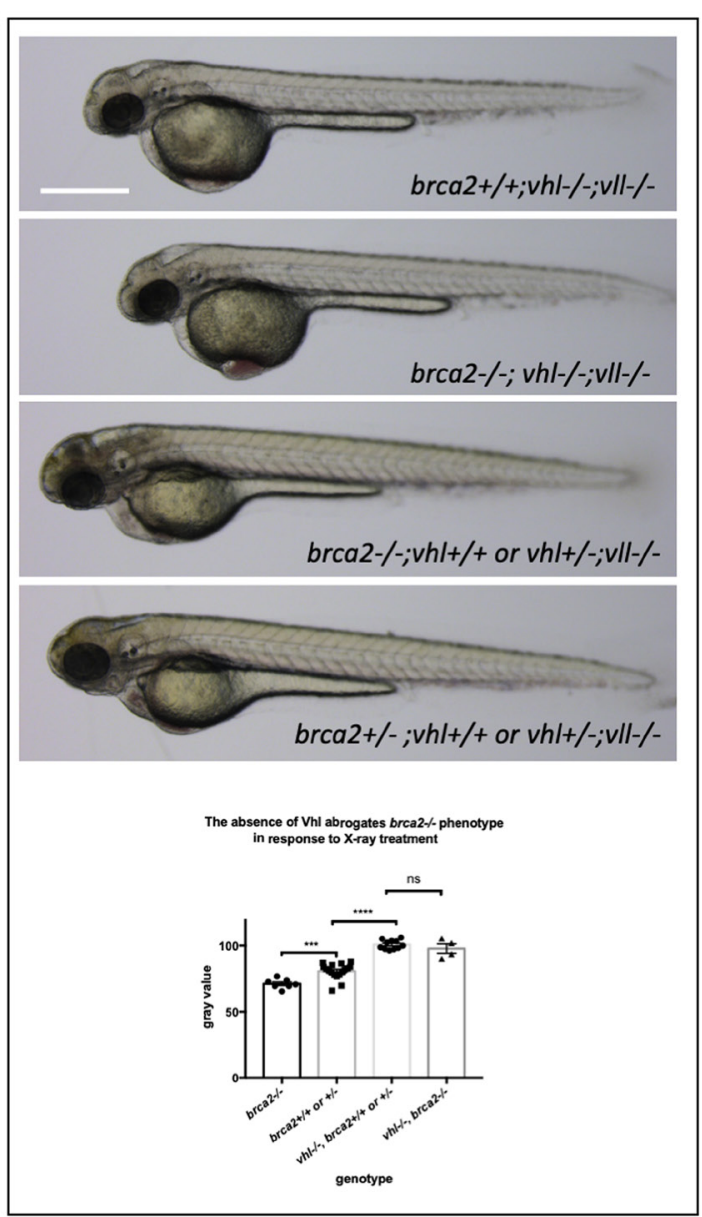

Figure 10: The elevated Hif level in the vhl-/- mutants alleviates cell deaths induced by the mutation in brca2. (A) brca2-/- embryos were more sensitive to X-ray treatment in comparison to their siblings demonstrating the increased cell death in CNS. ${ }^{* * * *} p<0.0001$, unpaired $t$-test. (B) The Acridine Orange staining confirmed the increased apoptosis in the $b r c a 2-/-$ embryos. ${ }^{* * *} p<0.001$, unpaired $t$-test. (C) X-ray treatment induced a few EGFP positive cells in the brca2-/- embryos, indicating the loss of both $v h l$ alleles in these embryos. On the other hand the EGFP positive cells were hardly observed in their siblings. The Hif activator treatment suppressed the appearance of EGFP positive $v h l$ mutant cells in the $b r c a 2-/-$ embryos. ${ }^{* * *} p<0.001$, one way AVOVA. (D) brca2-/- embryos were more sensitive to X-ray treatment in comparison to their siblings. However, when the $v h l-/-; v l l-/-$ mutations are introduced in the brca2-/embryos, increased apoptosis in the CNS of brca2-/- embryos was suppressed and the brca2-/- embryos were indistinguishable from their siblings. ${ }^{* * *} p<0.001,{ }^{* * * *} p<0.0001,{ }^{\text {ns }} p>0.05$, one way ANOVA. Scale bars: $0.5 \mathrm{~mm}$. 
DNA damage and apoptosis. On the contrary, the weak upregulation of Hif in the vll single mutants was not sufficient to suppress the DNA repair defect caused by the lack of Vll function in DNA repair, manifesting the increased $\mathrm{LOH}$ in the $v h l$ locus in response to ionizing irradiation.
Our data suggest that zebrafish VHL homologues play two opposing roles. On the one hand, they suppress DNA repair by suppressing Hif; on the other, $v l l$ promotes DNA repair. Therefore, we speculate that the balance of these two opposing roles of VHL might be associated with cancer initiation. HIF activation might initially be able to
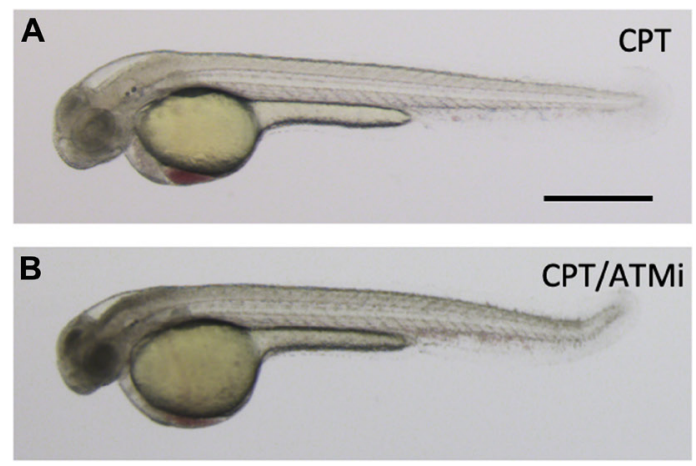

C

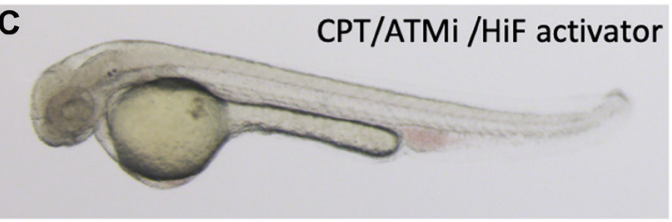

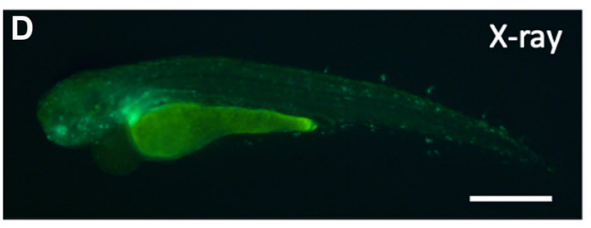
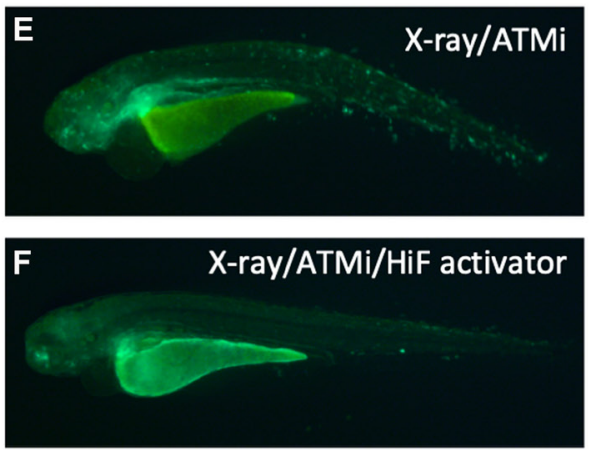

G

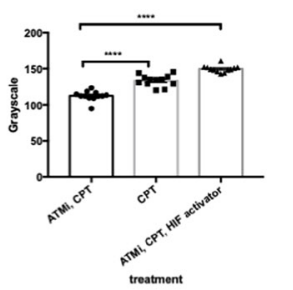

H

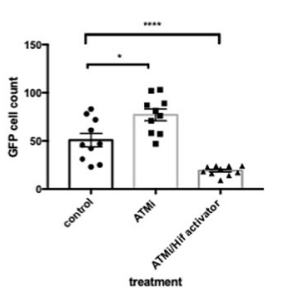

Figure 11: Hif activation abolishes the sensitivity to CPT induced cell death in ATM inhibitor treated embryos. (A) The wild type embryos were treated with $10 \mathrm{nM} \mathrm{CPT}$ to induce cell death in the CNS. (B) When the embryos were treated with ATMi before CPT treatment, the embryos were highly sensitised to CPT treatment and induced severe cell death in the CNS. (C) When the embryos were treated with Hif activator in combination with ATMi prior to CPT treatment, the embryos were well protected from CPT/ATMi induced cell death in CNS. The quantification of the data is shown in $(\mathbf{G})^{* * * *} p<0.0001$, one way ANOVA. (D) The $v h l+/-; v l l-/-; T g(p h d 3:: E G F P)^{i l 44}$ embryos were treated with X-ray to induce LOH at the $v h l$ locus. (E) When the $v h l+/-; v l l-/-; T g$ (phd $3:$ EGFP) ${ }^{i l 44}$ embryos were treated with ATMi before the X-ray treatment, there was a dramatic increase in the LOH in the $v h l$ locus in the ATMi treated embryos. (F) When the embryos were treated with Hif activator in combination with ATMi before the X-ray treatment, there was a remarkable reduction in the LOH in the $v h l$ locus. The quantification of the data is shown in $(\mathbf{H}){ }^{*} p<0.05,{ }^{* * * *} p<0.0001$, one way ANOVA. Scale bars: $0.5 \mathrm{~mm}$.

MRC5

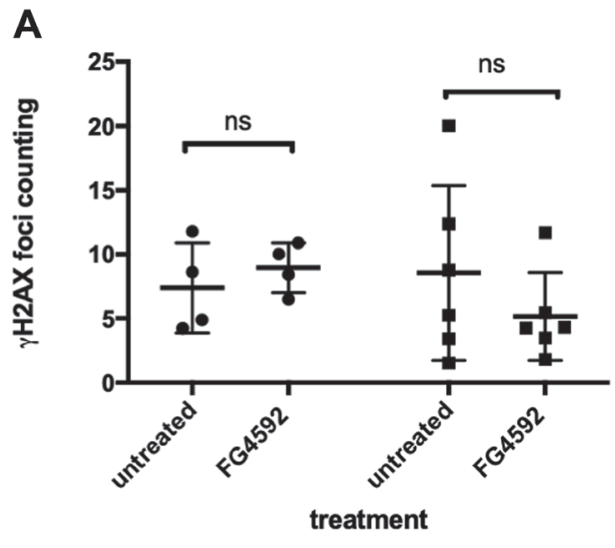

U2OS cells

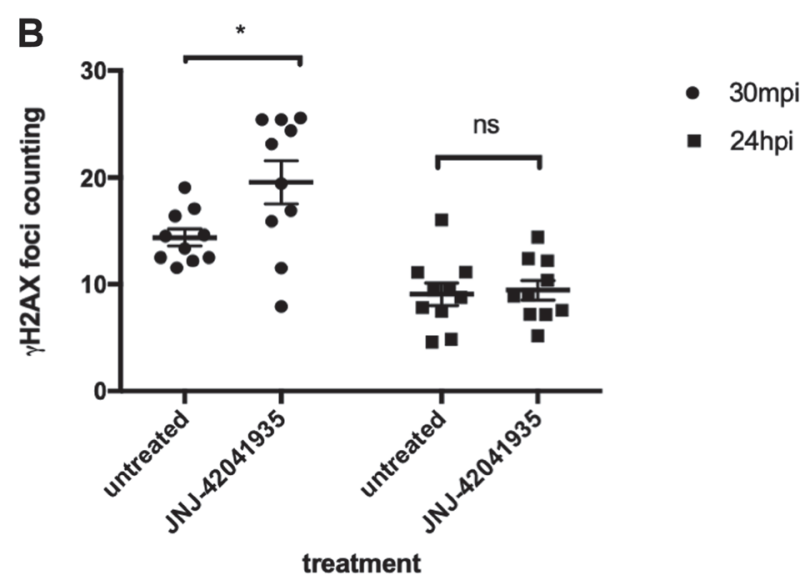

Figure 12: HIF activation does not protect mammalian cells from X-ray induced DNA damage. (A) We treated MRC5 cells with HIF activator FG 4592 for 3 hours prior to X-ray treatment at 5 gray. The cells were fixed at 4 hpi and 24 hpi with $4 \%$ PFA and stained with $\gamma \mathrm{H} 2 \mathrm{AX}$. This revealed that there was no significant difference in the $\gamma \mathrm{H} 2 \mathrm{AX}$ formation between HIF activator treated cells and nontreated cells. ${ }^{\text {ns }} p>0.05$, one way ANOVA. (B) The cells were treated with HIF activator JNJ-42041935 for 3 hours prior to X-ray treatment at 2 gray. The cells were fixed at $30 \mathrm{mpi}$ and $24 \mathrm{hpi}$ and stained with $\gamma \mathrm{H} 2 \mathrm{AX}$. At both time points, we could not observe the reduction in $\gamma \mathrm{H} 2 \mathrm{AX}$ formation in the HIF activator treated group in comparison to untreated group. At $30 \mathrm{mpi}$, there was even an increase in the number of $\gamma \mathrm{H} 2 \mathrm{AX}$ foci in the HIF activator treated group in opposition to our expectation. ${ }^{\mathrm{ns}} p>0.05,{ }^{*} p<0.05$, one way ANOVA. 
"mask" effects of the loss of VHL on genomic stability, however when the malignant cells finally form, they will be protected by the strong antiapoptotic function of HIF. Our data is consistent with resistance to the chemo and radio therapies seen in later stage of ccRCC in human through HIF's role in DNA repair and suppression of apoptosis. We propose zebrafish $v$ hl-/-; vll-/- mutants as an excellent platform for the screening for drugs that can interfere with the protection that is conferred by Hif, and therefore overcome resistance.

We discovered that the level of p53 was downregulated in $v h l-/-; \quad v l l-/-$ double mutants in response to the ionizing irradiation in comparison to their siblings. In addition, when we injected embryos with p53 morpholino, the injected embryos were all protected from CPT induced apoptosis even in the presence of intact $v h l$, suggesting that the decreased level of p53 is responsible for the protection of embryos from apoptosis in the $v h l$ mutants, at least partially. This is consistent with in vitro studies in which the role of pVHL in the p53 activation has been demonstrated [37]. However, we propose that the downregulated p53 level in the zebrafish vhl mutants is due to the elevated Hif, rather than by the Hif independent role of $\mathrm{Vhl}$ in regulation of $\mathrm{p} 53$. Importantly we found that the downregulation of p53 did not affect the increased $\mathrm{LOH}$ in the $v l l$ mutants, signifying the fact that the role of
Hif is not simply in regulating the level of p53, but Hif has a genuine role in the DNA repair. The downregulation of p53 could be the consequence of reduction in DNA damage due to the function of Hif in DNA repair.

Most interestingly, Hif activation also suppressed the increased apoptosis and DNA damage induced by $\mathrm{X}$-ray and CPT treatment in the brca 2 mutant and in the embryos with deficient ATM function. Both BRCA2 and ATM play important roles in the DNA repair pathways in human and their mutations increase the risk of cancer development. In addition, one of the distinctive features of A-T syndrome caused by the deficient ATM function is the defective movement and coordination due to neurodegeneration in cerebellum [47]. Similarly, the mutations in genes that encode proteins in the DNA repair machineries often lead to congenital neurodegenerative disorders in human, highlighting the importance of maintaining the genomic stability in the nervous system. For example, mutations in MRE11, TDP1 and aprataxin (APTX) result in neurodegenerative disorders such as A-T like disease (ATLD) [48], spinocerebellar ataxia with axonal neuropathy (SCAN1) [49], and ataxiaoculomotor apraxia-1 $[50,51]$ respectively. The efficacy of Hif activators for the suppression of DNA damage and apoptosis in brca 2 mutants and ATM inhibitor treated embryos in our study suggests that HIF activators might

\section{Clonogenic assay}
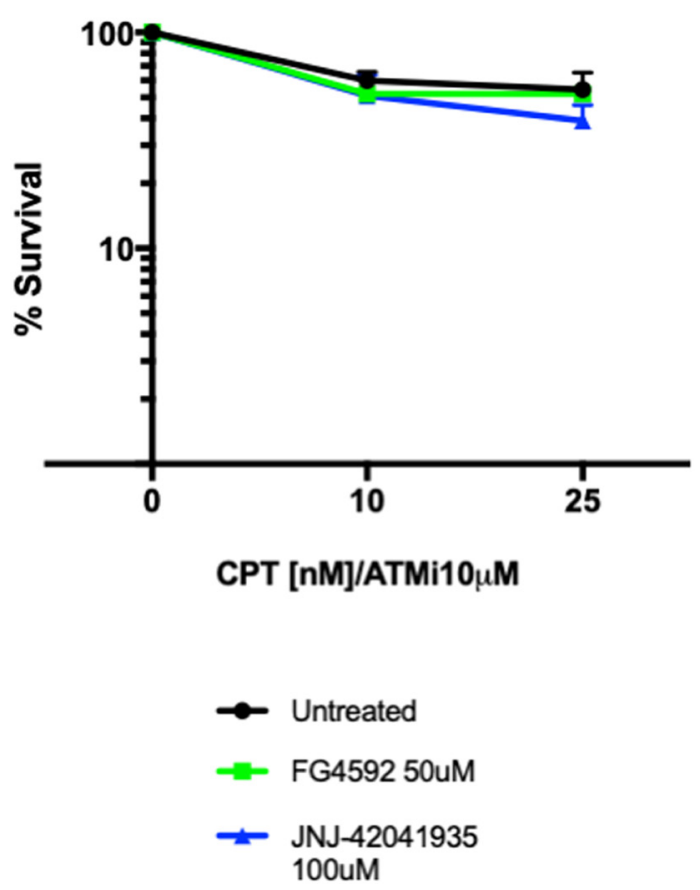

Figure 13: Hif activator treatment does not affect the survival of MRC5 cells. 4000 MRC5 cells were seeded in 6 well plates and left to grow overnight. The next day, the cells were treated with HIF activator (FG4592 or JNJ-42041935) and ATMi for 3 hours. CPT was added at either $10 \mathrm{nM}$ or $25 \mathrm{nM}$ concentration for 1 hour and the drugs were washed off. The colony formation was examined 7 days after the drug treatment. CPT/ATMi treatment decreased the survival rates but we could not find any effect of HIF activator on the survival of MRC5 cells. ${ }^{\text {ns }} p>0.05$, unpaired $t$-test. 
provide a possible intervention to slow the progression of such congenital neurodegenerative disorders.

Unfortunately, we could not reproduce the data we generated in the zebrafish in MRC5 and U2OS cells; HIF activation did not protect cells from X-ray induced DNA damage in these cell lines. HIF activator treatment did not affect the survival rates of cells after CPT treatment in the MRC5 cells either. We cannot currently explain why this is the case. We wanted to use the cell lines to gain mechanistic insight with regard to the particular DNA lesions and specific DDR proteins that are affected by HIF activation, taking advantage of abundant resources of antibodies which are available in the cell culture system. Nevertheless, our in vivo data are very clear, and it is perhaps an illustration of the importance of using in vivo systems to complement cell culture work. When cells are taken in culture and expanded, there is a strong and continuous selection for cells that can resist apoptosis and divide rapidly, thus cultured cells may have lost relevant characteristics (e. g. appropriate p53 activity) that are present in vivo. Perhaps the use of primary cells may overcome these issues. Currently, the limitation of our study is the lack of mechanistic understanding. We are left to raise questions: Against what specific DNA lesions does Hif activation protect? Are there any other targets of Hif than p53 that provide survival advantages to the cells lacking the functional Vhl? Given time, we could use mutants and treatments that lead to defects in various DNA repair pathways, and study how Hif activation can modulate the response such genotoxic stressors.

In summary, we discovered that HIF regulation and DNA repair role of human VHL are conserved in zebrafish $v h l$ and $v l l$. The activated Hif in the vhl; vll mutants strongly suppresses DNA damage and apoptosis induced by genotoxic stress. We speculate this could parallel resistance to chemo- and radio therapies in ccRCC, and propose zebrafish $v h l-/-; v l l-/-$ double mutants as a powerful model for the development of therapeutic reagents to overcome the resistance of ccRCC to chemoand radio therapies. We found Hif activation suppresses the DNA damage in the brca 2 and $v l l$ mutants and ATM deficient embryos and also prevents apoptosis. Currently at least one HIF activator is in the phase III clinical trials for the treatment of anemia $[52,53]$. Our results suggest that clinical benefits of HIF activation could expand beyond treatment of anemia, to that of disorders linked with DNA repair deficiency, like Ataxia Telangiectasia.

\section{MATERIALS AND METHODS}

\section{Zebrafish husbandry}

All the zebrafish strains used in this paper are maintained on $10 / 14$ hours dark/light cycle at $28^{\circ} \mathrm{C}$ according to the UK home office regulation at the University of Sheffield.

\section{Mutagenesis}

We generated $v l l$ mutants by zinc finger nucleases (ZFNs) purchased from Sigma-Sangamo Biosciences. A pair of ZFNs designed to bind the following sequences (in capitals) showed good activity when RNA encoding these was injected into embryos, and the efficiency of them was assayed by the loss of NcoI site (underlined) that covers the start ATG (bold) of $v l l$. caCAGCTCATCCATGGtgcaGAGCATCTGATGGAGct brca 2 mutants were induced by CRISPR/Cas9 system. The sequence of gRNA targeting brca2 is as follow. 5'TGGCTTGGATATGACACACC 3' The brca2 mutant allele, brca $2^{\text {sh } 515}$, contains an $83 \mathrm{bp}$ insertion at the amino acid 445 (exon 10) position introducing premature stop.

\section{Cell culture}

MRC5 and U2OS cells were grown in Minimum Essential Media (MEM; Sigma-Aldrich) supplemented with $10 \%$ fetal bovine serum (Sigma-Aldrich), 2\% L-glutamine (Sigma-Aldrich).

\section{Ionizing irradiation treatment in zebrafish}

Zebrafish embryos were irradiated at 20 Gray at $1 \mathrm{dpf}$ or $2 \mathrm{dpf}$ with $\gamma$-ray using the irradiator (CIS bio international) at the Royal Hallamshire Hospital animal facility or with X-ray using the irradiator (Faxitron X-ray Corporation) at the Biomedical Science Department at the University of Sheffield.

\section{Injections into zebrafish embryos}

CRISPR against $v h l$ and $A R$ was injected into the newly fertilized 1-cells stage embryos using microinjector (Pneumatic PicoPump WPI) at 100 $\mathrm{uM}$ concentration at $1 \mathrm{nl}$ volume. Target sequences were CCGCACGAGCAGTGGTACCC (ar) and CAGCCGTACGTGAACATTCC $(v h l)$. Image analysis to determine LOH levels in $v h l+/-; \operatorname{Tg}$ (phd3:: EGFP) ${ }^{i 144}$ vs vhl+/-; vll-/-; $\operatorname{Tg}$ (phd3:: EGFP)il44 embryos was performed as illustrated in Supplementary Figure 7.

\section{Deep sequencing}

The embryos from $v h l+/-$ pair mating and $v h l+/-$; vll-/- pair mating were collected and the 15 embryos per each genotype were selected. After genomic DNAs were extracted from each group, the deep sequencing was performed with the primers shown below with three biological replicates.

Forward primers for AR:
TCGTCGGCAGCGTCAGATGTGTATAAGAGAC
AGCCT CCAAAG CAAAGG ACA CC (inner primer),
ATGCCCCGTGATCTGAATGA (outer primer),
Reverse primers for AR: GTCTCGTGGGCTCGGA


GATGTGTATAAGAGACAGAAA TCAGTTGTGGC GCAGAG (inner primer), ACCCTGTGCACGGTGTATTA (outer primer), Forward primers for vhl: TCGTCGGCAGCGTCAGATGTGTATAAGAGACAG

TGAAGCTTTAGTCTAACTCGGTG (inner primer), CACGAACCCACAAAAGTTGTTAT (outer primer),

Reverse primers for vhl: GTCTCGTGGGCTCG GAGATGTGTATAAGAGACAG AATTCAGCATAATTT CACGAACC (inner primer), CTTCACCGACTCT CACAAGATTA (outer primer). Analysis of mutations frequencies and sizes was performed using BATCH-GE [54, $55]$.

\section{Immunostaining in zebrafish}

Embryos were fixed at $-20^{\circ} \mathrm{C}$ with methanol: acetone (50\%:50\%). Embryos were then permeabilised with $1 \%$ triton for 1 hour. The embryos were subsequently incubated in the blocking solution (2\% Roche block, $5 \%$ calf serum, $1 \%$ DMSO). $\gamma \mathrm{H} 2 \mathrm{AX}$ antibody (GTX127342, GeneTex,) was treated at 1:1000 dilution and incubated overnight. Secondary antibody (Alexa Flore 488 or Alexa Flore 546, Molecular Probe) was treated at 1:1000 and the embryos were incubated for 2 hours at room temperature. After the washing, the embryos were visualized using a confocal microscope.

\section{Chemical treatment}

ATM inhibitor (KU-55933, Sigma-Aldrich) was treated both in zebrafish and cell cultures at $10 \mathrm{uM}$ concentration. HIF activators (FG 4592, Stratech or JNJ42041935, Johnson and Johnson) were treated at 15-50 $\mathrm{uM}$ and $100 \mathrm{uM}$ respectively in zebrafish and in cell cultures. CPT ((S)-(+)-Camptothecin, Sigma Aldrich) was treated at $10-25 \mathrm{nM}$ concentration in zebrafish and in cell cultures as indicated in the text.

\section{TUNEL staining}

3 hours after the irradiation with X-ray, the embryos were fixed with $4 \%$ PFA overnight at $4^{\circ} \mathrm{C}$. The embryos were washed 3 times for 5 minutes with PBS and permeabilised with $10 \mu \mathrm{g} / \mathrm{ml}$ proteinase $\mathrm{K}$ for 20 minutes. The fixed embryos were then washed and stained using the ApopTag Red in situ apoptosis detection kit (Chemicon).

\section{q-RCR for full length and short form of p53}

The embryos from $v h l+/-$ pair mating and $v h l+/-$; $v l l-/-$ pair mating were collected and raised to $2 \mathrm{dpf}$. Then the embryos were irradiated with $\gamma$ - ray at 20 gray dose. The embryos were pools according to their genotype and Total RNA was extracted. The specific TaqMan probes for full length and short form of $\mathrm{p} 53$ were designed and qPCR was performed after the first strand cDNA synthesis.

\section{Western analysis}

40 embryos were collected for each sample. The yolk was removed from the embryos with $1 \mathrm{ml}$ of icecold PBS by pipetting up and down with yellow tip several times. The $50 \mathrm{ul}$ of lysis buffer $(200 \mathrm{mM}$ Hepes, $40 \mathrm{mM}$ $\mathrm{NaCl}, 2 \mathrm{mM} \mathrm{MgCl} 2,0.5 \%$ Triton X-100, $1 \times$ protease inhibitor (Roche, 4693159001), $1 \times$ phosphatase inhibitor (Roche, 4906837001), $25 \mathrm{U} / \mathrm{mL}$ BaseMuncher (Expedeon, BM0025)) was added to each sample, which was followed by 30 minute incubation on ice. Then the samples were centrifuged for 15 minutes at $4^{\circ} \mathrm{C}$, and the lysate was transferred into fresh Eppendorf tubes. The amount of protein was quantified in each sample by Bradford assay in Jenway visible spectrophotometer (Genova, 6320D). $40 \mathrm{ug}$ of protein for each sample was mixed with $5 \mathrm{xPLB}$ (protein loading buffer) and $\mathrm{H}_{2} \mathrm{O}$ to make up the final volume of $25 \mathrm{ul}$. The samples were denatured at $95^{\circ} \mathrm{C}$ for 5 minutes and loaded on the $4-15 \%$ precast polyacrylamide gel (Bio-rad 4561983). The gel was then transferred onto the nitrocellulose membrane (Bio-Rad, 170-4271) using the Trans-Blot Turbo ${ }^{\circledR}$ transfer ${ }^{\mathrm{TM}}$ system (Bio-Rad, 17001915). The membrane was blocked in 5\% milk/TBST (200 mM Tris, $140 \mathrm{mM} \mathrm{NaCl}, 0.1 \%$ Tween-20, pH 7.4) for 1 hour and incubated in p53 antibody [38] at 1:200 dilution overnight at $4^{\circ} \mathrm{C}$. Next day the membrane was washed for 5 minutes 3 times in TBST, and incubated in the secondary antibody (anti-mouse IgG $(\mathrm{H}+\mathrm{L})$-HRP conjugate (Bio-Rad (170-6516)) at 1:4000 dilution for 1 hour. After 5 minute wash 3 times, Luminol/enhancer and peroxide reagents (Bio-Rad, 1705060) were added onto the membrane at 1:1 ratio and the staining was visualised in the ChemiDoc MP imaging system (Bio-Rad, 1708280).

\section{Acridine orange staining}

Acridine Orange (A6014, Sigma Aldrich) was prepared at $1 \mathrm{mg} / \mathrm{ml}(\times 100$, stock solution $)$ in miliQ water, and stored at $-20^{\circ} \mathrm{C}$. The embryos were incubated in $\times 1$ Acridine Orange for 30 minutes in E3 fish medium. The embryos were washed three times each for 10 minutes.

\section{Immunostaining in the MRC5 and U2OS cells}

70,000 of either MRC5 cells or U2OS cells were seeded onto the cover slips in the 24 well plates, and the cells were grown overnight at $37^{\circ} \mathrm{C}$. Then the cells were treated with HIF activator for 3 hours followed by $\mathrm{x}$-ray treatment with a dose of either 5 or 2 gray dose. The irradiated cells were fixed with 4\% PFA for 10 minutes after 30 minutes, 4 hours and 24 hours irradiation. The fixed cells were permeabilized with $0.2 \%$ tween and blocked for 1 hour with $3 \%$ BSA. The primary antibody gH2AX (mouse JWB301-Milipore) was added at 1:500 dilution and the cells were incubated in the primary antibody overnight at $4^{\circ} \mathrm{C}$. Next day, anti-mouse Alexa Flour 594 (Invitrogen A11005) was added and the cells 
were incubated in the secondary antibody for one hour. The cells were counter stained with DAPI before mounting with mounting solution.

\section{Clonogenic studies}

4000 cells per well were plated into the $10 \mathrm{~cm}$ plates. After leaving the cells to grow overnight at $37^{\circ} \mathrm{C}$, cells were treated with chemicals, either ATMi at $10 \mathrm{uM}$ concentration alone or ATMi and HIF activators JNJ-42041935 at $100 \mathrm{uM}$ or FG 4592 at $50 \mathrm{uM}$ and incubated at $37^{\circ} \mathrm{C}$ for 3 hours. Next CPT was added to the wells at $10 \mathrm{nM}$ or $25 \mathrm{nM}$ concentration, and cells were incubated 1 hour. The drugs were poured off and the fresh media were added to the plates. The cells were then returned to $37^{\circ} \mathrm{C}$ incubator for another 7 days for the colony formation. When the colonies are formed, the media were poured off and cells were fixed with $80 \%$ ethanol. After the cells were left to dry, they were stained with $1 \%$ methylene blue for 1 hour. Finally the plates were rinsed with water and colonies were counted.

\section{Statistical analysis}

The data were analysed using the GraphPad program. All data were presented with the mean value of s. e. m. Statistical difference was analysed by the student's $t$-test for two groups of data, one-way ANOVA for more than two groups. Two-way ANOVA test was performed to determine the effect of X-rays and mutations on the expression levels of p53 and $\Delta 113 \mathrm{p} 53$. The significance of Hif activation on the survival of zebrafish embryos was tested by chi-square test. $p<0.05$ was considered statistically significant.

\section{Imaging}

The embryos were visualized using Leica dissecting microscope and the images were captured using Leica Application System v4.9.0 software. The confocal images were acquired using Olympus FV1000 available at the light microscope facility at the Biomedical Science Department at the University of Sheffield.

\section{Abbreviations}

VHL: Von Hippel Lindau; ccRCC: clear cell renal cell carcinoma; HIF: Hypoxia Inducible Factor; PHD: Prolyl Hydroxylase; HRE: HIF responsive element; DSB: double strand break; HR: homologous recombination; A-T: Ataxia -Telangiectasia; MMR: mismatch repair; LOH: loss of heterozygosity; TUNEL: terminal deoxynucleotidyl transferase dUTP nick end labelling; CPT: Camptothecin; CNS: central nervous system; ATM: ATM serine/threonine kinase; ATMi: ATM inhibitor; mpi: minutes post irradiation; APTX: aprataxin; ATLD: A-T like disease; SCAN1: spinocerebellar ataxia with axonal neuropathy; ZFN: zinc finger nucleases.

\section{Author contributions}

Kim HR, wrote the paper, designed and executed experiments. Santhakumar K, wrote the paper, designed and executed experiments. Markham E, executed experiments. Baldera D, executed experiments. Greenald $\mathrm{D}$, carried out qPCR analysis. Bryant HE, designed experiments and commented on manuscript. ElKhamisy SF, designed experiments and commented on manuscript. van Eeden FJ, designed experiments, executed experiments, wrote the manuscript.

\section{ACKNOWLEDGMENTS}

We thank Dan Harris and Dr Shih-Chieh Chiang for help with cell culture work. The Bateson Centre aquaria staff kindly provided care for zebrafish. We also would like to thank Elisabeth Kugler for help with $\gamma \mathrm{H} 2 \mathrm{AX}$ foci counting script and Dr Phil Elks for providing us with the dominant active form of Hif constructs. We are also very grateful to professor Jinrong Peng for providing us with p53 antibody.

\section{CONFLICTS OF INTEREST}

None.

\section{FUNDING}

The work was supported by two BBSRC Grants: BB/R015457/1 and BB/M02332X/1.

\section{REFERENCES}

1. Gossage L, Eisen T, Maher ER. VHL, the story of a tumour suppressor gene. Nat Rev Cancer. 2015; 15:55-64. https:// doi.org/10.1038/nrc3844. [PubMed]

2. Maher ER, Neumann HP, Richard S. von Hippel-Lindau disease: a clinical and scientific review. Eur J Hum Genet. 2011; 19:617-623. https://doi.org/10.1038/ejhg.2010.175. [PubMed]

3. Jaakkola P, Mole DR, Tian YM, Wilson MI, Gielbert J, Gaskell SJ, von Kriegsheim A, Hebestreit HF, Mukherji M, Schofield CJ, Maxwell PH, Pugh CW, Ratcliffe PJ. Targeting of HIF-alpha to the von HippelLindau ubiquitylation complex by O2-regulated prolyl hydroxylation. Science. 2001; 292:468-472. https://doi. org/10.1126/science.1059796. [PubMed]

4. Kaelin WG Jr. The VHL Tumor Suppressor Gene: Insights into Oxygen Sensing and Cancer. Trans Am Clin Climatol Assoc. 2017; 128:298-307. [PubMed] 
5. Semenza GL. Hypoxia-inducible factors in physiology and medicine. Cell. 2012; 148:399-408. https://doi. org/10.1016/j.cell.2012.01.021. [ubMed]

6. Li M, Kim WY. Two sides to every story: the HIFdependent and HIF-independent functions of pVHL. J Cell Mol Med. 2011; 15:187-195. https://doi.org/10.1111/ j.1582-4934.2010.01238.x. [PubMed]

7. Metcalf JL, Bradshaw PS, Komosa M, Greer SN, Stephen Meyn M, Ohh M. K63-ubiquitylation of VHL by SOCS1 mediates DNA double-strand break repair. Oncogene. 2014; 33:1055-1065. https://doi.org/10.1038/onc.2013.22. [PubMed]

8. Scanlon SE, Hegan DC, Sulkowski PL, Glazer PM. Suppression of homology-dependent DNA double-strand break repair induces PARP inhibitor sensitivity in VHLdeficient human renal cell carcinoma. Oncotarget. 2017; 9:4647-4660. https://doi.org/10.18632/oncotarget.23470. [PubMed]

9. Hoffman MA, Ohh M, Yang H, Klco JM, Ivan M, Kaelin WG Jr. von Hippel-Lindau protein mutants linked to type 2C VHL disease preserve the ability to downregulate HIF. Hum Mol Genet. 2001; 10:1019-1027. https://doi. org/10.1093/hmg/10.10.1019. [PubMed]

10. Morris MR, Hughes DJ, Tian YM, Ricketts CJ, Lau KW, Gentle D, Shuib S, Serrano-Fernandez P, Lubinski J, Wiesener MS, Pugh CW, Latif F, Ratcliffe PJ, et al. Mutation analysis of hypoxia-inducible factors HIF1A and HIF2A in renal cell carcinoma. Anticancer Res. 2009; 29:4337-4343. [PubMed]

11. Fu L, Minton DR, Zhang T, Nanus DM, Gudas LJ. GenomeWide Profiling of TRACK Kidneys Shows Similarity to the Human ccRCC Transcriptome. Mol Cancer Res. 2015; 13:870-878. https://doi.org/10.1158/1541-7786.MCR-140423. [PubMed]

12. Gudas LJ, Fu L, Minton DR, Mongan NP, Nanus DM. The role of HIF1alpha in renal cell carcinoma tumorigenesis. J Mol Med (Berl). 2014; 92:825-836. https://doi.org/10.1007/ s00109-014-1180-z. [PubMed]

13. Fu L, Wang G, Shevchuk MM, Nanus DM, Gudas LJ. Generation of a mouse model of Von Hippel-Lindau kidney disease leading to renal cancers by expression of a constitutively active mutant of HIF1alpha. Cancer Res. 2011; 71:6848-6856. https://doi.org/10.1158/0008-5472. CAN-11-1745. [PubMed]

14. Percy MJ, Furlow PW, Lucas GS, Li X, Lappin TR, McMullin MF, Lee FS. A gain-of-function mutation in the HIF2A gene in familial erythrocytosis. N Engl J Med. 2008; 358:162-168. https://doi.org/10.1056/NEJMoa073123. [PubMed]

15. Ang SO, Chen H, Hirota K, Gordeuk VR, Jelinek J, Guan Y, Liu E, Sergueeva AI, Miasnikova GY, Mole D, Maxwell PH, Stockton DW, Semenza GL, et al. Disruption of oxygen homeostasis underlies congenital Chuvash polycythemia. Nat Genet. 2002; 32:614-621. https://doi.org/10.1038/ ng1019. [PubMed]
16. Lopez-Otin C, Blasco MA, Partridge L, Serrano M, Kroemer G. The hallmarks of aging. Cell. 2013; 153:11941217. https://doi.org/10.1016/i.cell.2013.05.039. [PubMed]

17. Menck CF, Munford V. DNA repair diseases: What do they tell us about cancer and aging? Genet Mol Biol. 2014; 37:220 233. https://doi.org/10.1590/S1415-47572014000200008. [PubMed]

18. Spry M, Scott T, Pierce H, D'Orazio JA. DNA repair pathways and hereditary cancer susceptibility syndromes. Front Biosci. 2007; 12:4191-4207. https://doi. org/10.2741/2380. [PubMed]

19. Gatti R, Perlman S. Ataxia-Telangiectasia. In: Adam MP, Ardinger HH, Pagon RA, Wallace SE, Bean LJH, Stephens K, Amemiya A, eds. GeneReviews((R)). Seattle, WA. 1993. https://doi.org/10.1007/978-3-642-78278-7. [PubMed]

20. Bryant HE, Schultz N, Thomas HD, Parker KM, Flower D, Lopez E, Kyle S, Meuth M, Curtin NJ, Helleday T. Specific killing of BRCA2-deficient tumours with inhibitors of poly(ADP-ribose) polymerase. Nature. 2005; 434:913-917. https://doi.org/10.1038/nature03443. [PubMed]

21. Borrell B. How accurate are cancer cell lines? Nature. 2010; 463:858. https://doi.org/10.1038/463858a. [PubMed]

22. Wilding JL, Bodmer WF. Cancer cell lines for drug discovery and development. Cancer Res. 2014; 74:2377-2384. https:// doi.org/10.1158/0008-5472.CAN-13-2971. [PubMed]

23. Xia Y, Jiang L, Zhong T. The role of HIF-1 $\alpha$ in chemo-/ radioresistant tumors. Onco Targets Ther. 2018; 11:30033011. https://doi.org/10.2147/OTT.S158206. [PubMed]

24. Calvo-Asensio I, Dillon ET, Lowndes NF, Ceredig R. The Transcription Factor Hif-1 Enhances the Radio-Resistance of Mouse MSCs. Front Physiol. 2018; 9:439. https://doi. org/10.3389/fphys.2018.00439. [PubMed]

25. Lu C, El-Deiry WS. Targeting p53 for enhanced radio- and chemo-sensitivity. Apoptosis. 2009; 14:597-606. https:// doi.org/10.1007/s10495-009-0330-1. [PubMed]

26. Howe K, Clark MD, Torroja CF, Torrance J, Berthelot C, Muffato M, Collins JE, Humphray S, McLaren K, Matthews L, McLaren S, Sealy I, Caccamo M, et al. The zebrafish reference genome sequence and its relationship to the human genome. Nature. 2013; 496:498-503. https://doi. org/10.1038/nature12111. [PubMed]

27. van Rooijen E, Voest EE, Logister I, Korving J, Schwerte T, Schulte-Merker S, Giles RH, van Eeden FJ. Zebrafish mutants in the von Hippel-Lindau tumor suppressor display a hypoxic response and recapitulate key aspects of Chuvash polycythemia. Blood. 2009; 113:6449-6460. https://doi. org/10.1182/blood-2008-07-167890. [PubMed]

28. van Rooijen E, Voest EE, Logister I, Bussmann J, Korving J, van Eeden FJ, Giles RH, Schulte-Merker S. von Hippel-Lindau tumor suppressor mutants faithfully model pathological hypoxia-driven angiogenesis and vascular retinopathies in zebrafish. Dis Model Mech. 2010; 3:343353. https://doi.org/10.1242/dmm.004036. [PubMed] 
29. Kim HR, Greenald D, Vettori A, Markham E, Santhakumar $\mathrm{K}$, Argenton F, van Eeden F. Zebrafish as a model for von Hippel Lindau and hypoxia-inducible factor signaling. Methods Cell Biol. 2017; 138:497-523. https://doi. org/10.1016/bs.mcb.2016.07.001. [PubMed]

30. Santhakumar K, Judson EC, Elks PM, McKee S, Elworthy S, van Rooijen E, Walmsley SS, Renshaw SA, Cross SS, van Eeden FJ. A zebrafish model to study and therapeutically manipulate hypoxia signaling in tumorigenesis. Cancer Res. 2012; 72:4017-4027. https://doi.org/10.1158/0008-5472. CAN-11-3148. [PubMed]

31. Greenald D, Jeyakani J, Pelster B, Sealy I, Mathavan S, van Eeden FJ. Genome-wide mapping of Hif-1alpha binding sites in zebrafish. BMC Genomics. 2015; 16:923. https:// doi.org/10.1186/s12864-015-2169-x. [PubMed]

32. Pescador N, Cuevas Y, Naranjo S, Alcaide M, Villar D, Landazuri MO, Del Peso L. Identification of a functional hypoxia-responsive element that regulates the expression of the egl nine homologue 3 (egln3/phd3) gene. Biochem J. 2005; 390:189-197. https://doi.org/10.1042/BJ20042121. [PubMed]

33. Pena-Llopis S, Christie A, Xie XJ, Brugarolas J. Cooperation and antagonism among cancer genes: the renal cancer paradigm. Cancer Res. 2013; 73:4173-4179. https:// doi.org/10.1158/0008-5472.CAN-13-0360. [PubMed]

34. Ryan AJ, Squires S, Strutt HL, Johnson RT. Camptothecin cytotoxicity in mammalian cells is associated with the induction of persistent double strand breaks in replicating DNA. Nucleic Acids Res. 1991; 19:3295-3300. https://doi. org/10.1093/nar/19.12.3295. [PubMed]

35. Ashour ME, Atteya R, El-Khamisy SF. Topoisomerasemediated chromosomal break repair: an emerging player in many games. Nat Rev Cancer. 2015; 15:137-151. https:// doi.org/10.1038/nrc3892. [PubMed]

36. Liao C, Beveridge R, Hudson JJR, Parker JD, Chiang SC, Ray S, Ashour ME, Sudbery I, Dickman MJ, ElKhamisy SF. UCHL3 Regulates Topoisomerase-Induced Chromosomal Break Repair by Controlling TDP1 Proteostasis. Cell Reports. 2018; 23:3352-3365. https://doi. org/10.1016/j.celrep.2018.05.033. [PubMed]

37. Roe JS, Youn HD. The positive regulation of $\mathrm{p} 53$ by the tumor suppressor VHL. Cell Cycle. 2006; 5:2054-2056. https://doi.org/10.4161/cc.5.18.3247. [PubMed]

38. Gong L, Gong H, Pan X, Chang C, Ou Z, Ye S, Yin L, Yang L, Tao T, Zhang Z, Liu C, Lane DP, Peng J, et al. p53 isoform Delta113p53/Delta133p53 promotes DNA double-strand break repair to protect cell from death and senescence in response to DNA damage. Cell Res. 2015; 25:351-369. https://doi.org/10.1038/cr.2015.22. [PubMed]

39. Rodriguez-Mari A, Wilson C, Titus TA, Canestro C, BreMiller RA, Yan YL, Nanda I, Johnston A, Kanki JP, Gray EM, He X, Spitsbergen J, Schindler D, et al. Roles of brca2 (fancd1) in oocyte nuclear architecture, gametogenesis, gonad tumors, and genome stability in zebrafish. PLoS Genet. 2011; 7:e1001357. https://doi.org/10.1371/journal. pgen.1001357. [ [PubMed]
40. Shiloh Y, Ziv Y. The ATM protein kinase: regulating the cellular response to genotoxic stress, and more. Nat Rev Mol Cell Biol. 2013; 14:197-210. https://doi.org/10.1038/ nrm3546. [PubMed]

41. Smith K, Gunaratnam L, Morley M, Franovic A, Mekhail K, Lee S. Silencing of epidermal growth factor receptor suppresses hypoxia-inducible factor-2-driven VHL-/- renal cancer. Cancer Res. 2005; 65:5221-5230. https://doi. org/10.1158/0008-5472.CAN-05-0169. [PubMed]

42. Hughes MD, Kapllani E, Alexander AE, Burk RD, Schoenfeld AR. HIF-2alpha downregulation in the absence of functional VHL is not sufficient for renal cell differentiation. Cancer Cell Int. 2007; 7:13. https://doi. org/10.1186/1475-2867-7-13. [PubMed]

43. Nyhan MJ, O'Sullivan GC, McKenna SL. Role of the VHL (von Hippel-Lindau) gene in renal cancer: a multifunctional tumour suppressor. Biochem Soc Trans. 2008; 36:472-478. https://doi.org/10.1042/BST0360472. [PubMed]

44. Loeb LA. A mutator phenotype in cancer. Cancer Res. 2001; 61:3230-3239. [PubMed]

45. Loeb LA, Bielas JH, Beckman RA. Cancers exhibit a mutator phenotype: clinical implications. Cancer Res. 2008; 68:3551-3557. https://doi.org/10.1158/0008-5472.CAN-075835. [PubMed]

46. van den Bos H, Bakker B, Taudt A, Guryev V, ColomeTatche M, Lansdorp PM, Foijer F, Spierings DCJ. Quantification of Aneuploidy in Mammalian Systems. Methods Mol Biol. 2019; 1896:159-190. https://doi. org/10.1007/978-1-4939-8931-7_15. [PubMed]

47. Biton S, Barzilai A, Shiloh Y. The neurological phenotype of ataxia-telangiectasia: solving a persistent puzzle. DNA Repair (Amst). 2008; 7:1028-1038. https://doi. org/10.1016/j.dnarep.2008.03.006. [PubMed]

48. Stewart GS, Maser RS, Stankovic T, Bressan DA, Kaplan MI, Jaspers NG, Raams A, Byrd PJ, Petrini JH, Taylor AM. The DNA double-strand break repair gene hMRE11 is mutated in individuals with an ataxia-telangiectasia-like disorder. Cell. 1999; 99:577-587. https://doi.org/10.1016/ S0092-8674(00)81547-0. [PubMed]

49. Takashima H, Boerkoel CF, John J, Saifi GM, Salih MA, Armstrong D, Mao Y, Quiocho FA, Roa BB, Nakagawa M, Stockton DW, Lupski JR. Mutation of TDP1, encoding a topoisomerase I-dependent DNA damage repair enzyme, in spinocerebellar ataxia with axonal neuropathy. Nat Genet. 2002; 32:267-272. https://doi.org/10.1038/ng987. [PubMed]

50. Date H, Onodera O, Tanaka H, Iwabuchi K, Uekawa K, Igarashi S, Koike R, Hiroi T, Yuasa T, Awaya Y, Sakai T, Takahashi T, Nagatomo H, et al. Early-onset ataxia with ocular motor apraxia and hypoalbuminemia is caused by mutations in a new HIT superfamily gene. Nat Genet. 2001; 29:184-188. https://doi.org/10.1038/ng1001-184. [PubMed]

51. Moreira MC, Barbot C, Tachi N, Kozuka N, Uchida E, Gibson T, Mendonca P, Costa M, Barros J, Yanagisawa T, Watanabe M, Ikeda Y, Aoki M, et al. The gene mutated 
in ataxia-ocular apraxia 1 encodes the new HIT/Zn-finger protein aprataxin. Nat Genet. 2001; 29:189-193. https://doi. org/10.1038/ng1001-189. [PubMed]

52. Besarab A, Chernyavskaya E, Motylev I, Shutov E, Kumbar LM, Gurevich K, Chan DT, Leong R, Poole L, Zhong M, Saikali KG, Franco M, Hemmerich S, et al. Roxadustat (FG-4592): Correction of Anemia in Incident Dialysis Patients. J Am Soc Nephrol. 2016; 27:1225-1233. https:// doi.org/10.1681/ASN.2015030241. [PubMed]

53. Provenzano R, Besarab A, Sun CH, Diamond SA, Durham JH, Cangiano JL, Aiello JR, Novak JE, Lee T, Leong R, Roberts BK, Saikali KG, Hemmerich S, et al. Oral HypoxiaInducible Factor Prolyl Hydroxylase Inhibitor Roxadustat (FG-4592) for the Treatment of Anemia in Patients with CKD. Clin J Am Soc Nephrol. 2016; 11:982-991. https:// doi.org/10.2215/CJN.06890615. [PubMed]
54. Boel A, Steyaert W, De Rocker N, Menten B, Callewaert B, De Paepe A, Coucke P, Willaert A. BATCH-GE: Batch analysis of Next-Generation Sequencing data for genome editing assessment. Sci Rep. 2016; 6:30330. https://doi. org/10.1038/srep30330. [PubMed]

55. Boel A, Steyaert W, De Rocker N, Menten B, Callewaert B, De Paepe A, Coucke P, Willaert A. Publisher Correction: BATCH-GE: Batch analysis of Next-Generation Sequencing data for genome editing assessment. Sci Rep. 2018; 8:15845. https://doi.org/10.1038/s41598-01833869-y. [PubMed] 